\title{
mesure du fluage de la glace à l'aide du pressiomètre
}

\section{the creep of ice measured with the pressuremeter}

\author{
B.H KJARTANSON. \\ Atomic Energy Of Canada Limited ${ }^{*}$ \\ D.H. SHIELDS, L. DOMASCHUK \\ Civil Engineering Department, University of Manitoba* * \\ C.S. MAN \\ Department of Mathematics, University of Kentucky** *
}

Rev. Franç. Géotech. n 50 , pp. 23-38 (janvier 1990)

\section{Résumé}

Le pressiomètre permet de mesurer les paramètres du fluage de la glace in situ, c'est-à-dire de la glace en place, dans son environnement naturel, à la température et pour les contraintes auxquelles elle est alors exposée ; de plus, la glace ainsi testée n'est pas remaniée par échantillonnage ou manipulations. Afin de prouver que le pressiomètre peut, de fait, être utilisé pour mesurer les propriétés de fluage, toute une série d'essais au pressiomètre a été effectuée dans de la glace polycristalline à $-2{ }^{\circ} \mathrm{C}$ en laboratoire. Les observations suivantes ont été effectuées :

- le pressiomètre utilisé pour ces essais a permis de mesurer la déformation par fluage de manière précise pendant une période de 7 semaines ;

- les résultats des essais concordent, pour leur majeure partie, avec les résultats d'essais d'autres chercheurs ayant utilisé des essais de compression uniaxiale ;

- cette utilisation du pressiomètre remet en question au moins l'une des hypothèses les plus courantes concernant le fluage primaire,

\footnotetext{
Abstract

The pressuremeter has the potential to measure the creep parameters of ice in situ, that is to say in the field, in ice at its natural temperature and in its natural stress environment, and in ice which has not been disturbed by sampling and handling. In order to prove that the pressuremeter can in fact be used to measure creep properties, a series of pressuremeter tests was run in polycrystalline ice at $-2{ }^{\circ} \mathrm{C}$ in the laboratory. It was found that:

- the particular pressuremeter which was used maintained an ability to measure creep deformation accurately over a period of seven weeks;

- the test results agree for the most part with the findings of other investigators who used uniaxial compression tests ;

- the pressuremeter brings into question at least one of the more common assumptions regarding primary creep.
}

Une version anglaise de cet exposé a été publiée dans la Revue Canadienne de Géotechnique (Vol. 25, $n^{\circ} 2$ - Mai 1988).

- Pinawa, Manitoba.

Winnipeg, Manitoba.

*.. Lexington, Kentucky. 


\section{INTRODUCTION}

Depuis toujours, les propriétés du fluage de la glace ont été mesurées à partir d'essais de compression simple réalisés sur des éprouvettes cylindriques de glace artificielle. Dans le domaine du génie civil, il est cependant souvent nécessaire d'estimer le tassement d'une structure dont les fondations reposent sur de la glace (ou sur du pergélisol à haute teneur en glace) et donc les caractéristiques de cette glace naturelle (ou de ce pergélisol à haute teneur en glace). La démarche traditionnelle, qui consiste alors à soumettre des éprouvettes du matériau considéré à des essais de compression simple en laboratoire, se heurte aux obstacles traditionnels du remaniement lors du prélèvement et des modifications de température lors du transport vers le lieu d'essai.

Comme LADANYI et JOHNSTON P'ont souligné dès 1973, l'essai pressiométrique est parfaitement adapté à la mesure en place des caractéristiques de fluage des matériaux gelés. Des essais ont ainsi pu être effectués in situ dans de la glace (ou du pergélisol) non remaniée, soumise à des conditions naturelles de température et de contrainte.

Il existe de fait une différence notable entre les déformations auxquelles est soumis un matériau lors d'un essai de compression simple sur éprouvette cylindrique et au voisinage d'une sonde pressiométrique (BAGUELIN et al., 1978). Du fait de l'abondance de l'information disponible quant aux essais de compression sur glace et sol gelé, il nous apparaissait utile de comparer les caractéristiques de fluage déduites d'essais au pressiomètre avec celles provenant des essais de compression. La glace formée à partir d'eau douce étant plus facile à reconstituer qu'un pergélisol, il nous a semblé plus judicieux de nous intéresser en premier lieu à ce matériau, d'autant plus que son comportement avait fait l'objet de la majorité des résultats d'études en notre possession.

C'est ainsi qu'une série de 8 essais au pressiomètre, à un seul palier de chargement, a été réalisée sur 8 éprouvettes identiques de glace d'eau douce artificielle (KJARTANSON, 1986).

Tous les essais au pressiomètre ont été effectués dans de la glace maintenue à une température de $-2^{\circ} \mathrm{C}$, la température approximative du pergélisol se trouvant sous la mer de Beaufort, où des îles artificielles sont susceptibles d'être réalisées en vue de l'exploitation de champs de pétrole.

\section{FLUAGE DE LA GLACE A PARTIR D'ESSAIS DE COMPRESSION SIMPLE : SYNTHÈSE BIBLIOGRAPHIQUE}

\subsection{Relations :}

\section{"vitesse de déformation-temps" et « vitesse de déformation-déformation "}

Une synthèse excellente des connaissances disponibles sur le comportement de la glace à des températures négatives relativement élevées peut être faite, entre autre, à partir des travaux récents de MELLOR et COLE (1982 et 1983) et de JACKA (1984). La figure 1 (MELLOR et COLE, 1982) décrit le comportement au fluage de la glace en compression simple sous charge constante en fonction du temps. La figure 2 présente les mêmes données, mais dans un diagramme bilogarithmique, la déformation étant traduite en termes de vitesse de déformation (à partir de la pente des courbes de la figure 1).

Il apparaît clairement qu'il existe une vitesse de déformation minimale à un temps unique quel que soit le niveau donné de contrainte. Le temps correspondant aux vitesses de déformation minimale de la figure 2 est indiqué par des flèches sur les courbes de la figure 1: ces flèches correspondent aux points d'inflexion sur les différentes courbes de déformation en fonction du temps. Par convention, on considère que ces points d'inflexion correspondent généralement à la rupture, puisque au-delà de ces points, la vitesse de déformation ne cesse d'augmenter, ce qui traduit une accélération du phénomène de déformation.

Lorsque la vitesse de déformation est représentée en fonction de la déformation (au lieu du temps) on obtient la figure 3 . La rupture se fait à peu près à la même déformation dans les 9 essais, c'est-à-dire en moyenne à $0,82 \%$ de déformation ainsi que l'indique la droite verticale passant par les points correspondants. La position des flèches sur la figure 1 fait également apparaître cette dépendance vis-à-vis de la déformation. Les résultats obtenus par MELLOR et COLE sur 24 essais de compression sous charge constante appliquée sur de la glace à $-5^{\circ} \mathrm{C}$, et qui correspondent à une gamme de contraintes allant de 0,8 à $3,8 \mathrm{MPa}$, montrent clairement que la glace se rompt en compression à une valeur unique de la déformation, indépendante de la contrainte. Cette déformation movenne à la rupture est de $0.88 \%$ pour les 24 essais. D'autres chercheurs (SEGO et MORGENSTERN, 1983 ; JACKA, 1984) mentionnent également l'apparition de déformations correspondant à la rupture sous chargement pour des valeurs comprises entre 0.9 et $1.0 \%$, confirmant ainsi le chiffre de 0,9\% obtenu par MELLOR et COLE.

\subsection{Relations: vitesse de déformation et résistance}

Si des essais de compression sont effectués à une vitesse constante de déformation (comme ceux effectués par MELLOR et COLE en 1982, voir figure 4), par opposition à des essais à contrainte constante, la rupture (définie en tant que contrainte verticale maximale appliquée à l'éprouvette) correspond à environ $1 \%$ de déformation. La déformation moyenne à la rupture mentionnée par MELLOR et COLE pour 26 essais effectués à $-5^{\circ} \mathrm{C}$ était de $1,02 \%$; le point de rupture des courbes de la figure 4 se situe en moyenne à $1,17 \%$. Il existe donc une excellente concordance entre les résultats d'essais à contrainte constante et à vitesse de déformation constante dans le cas des essais de compression réalisés sur la glace. La figure 5 montre clairement que la contrainte à la rupture et la vitesse de déformation à la rupture (et dans une moindre mesure, la déformation à la rupture) peuvent être considérées cornme identiques, pour les applications pratiques, qu'elles aient été 


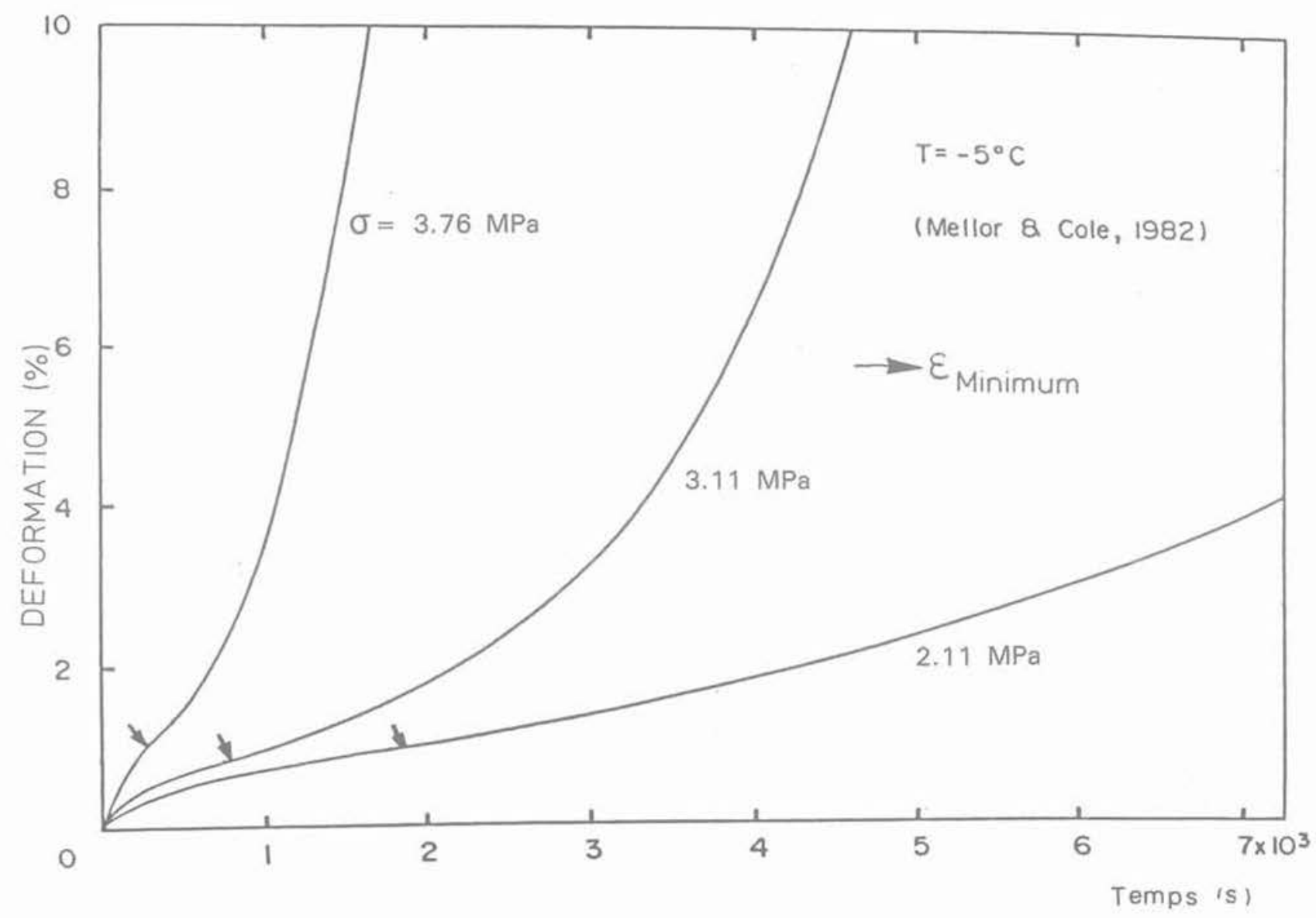

Fig. 1. - Fluage de la glace pure lors des essais de compression sous une contrainte constante. Fig. 1. - Creep of ice in compression under constant load.

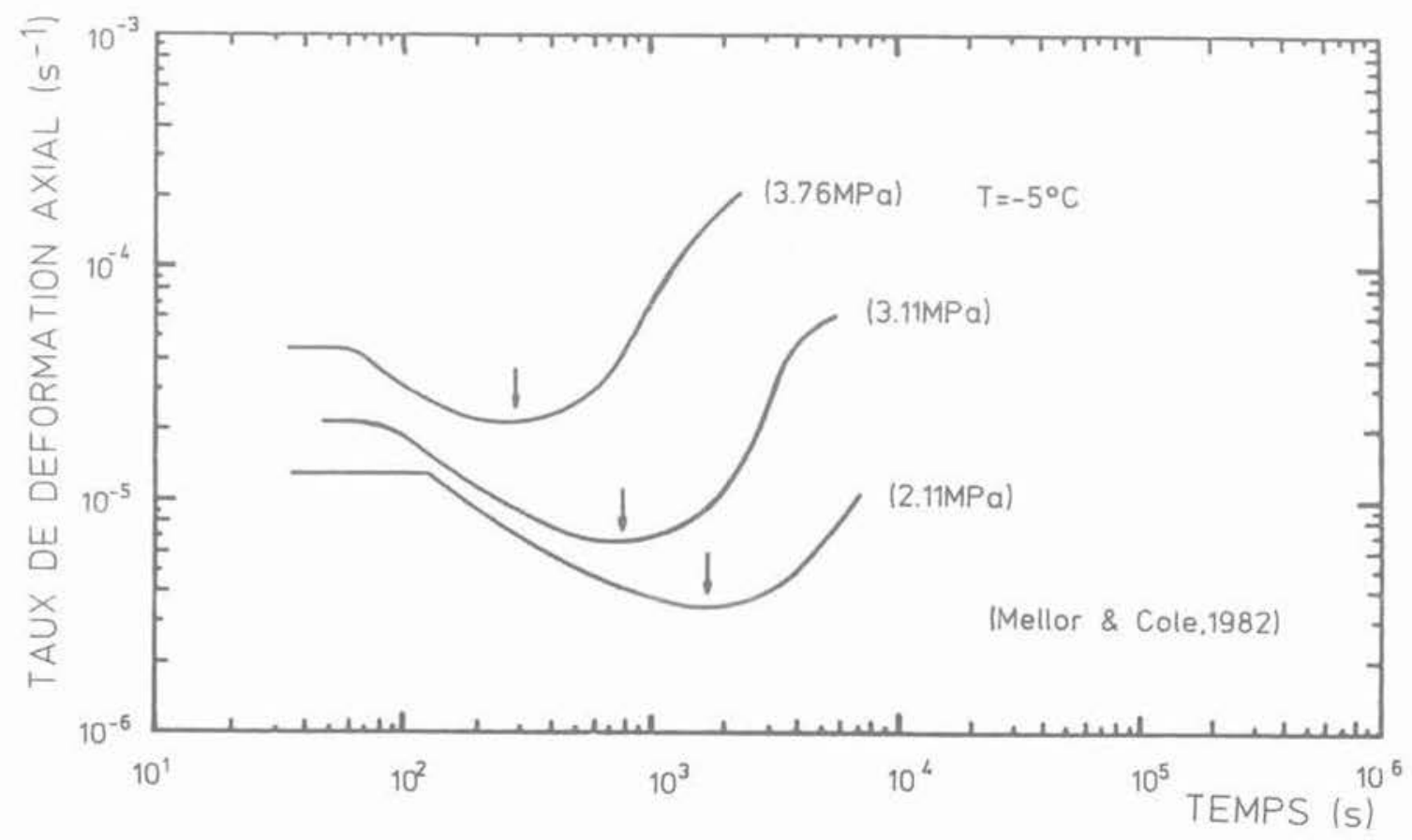

Fig. 2. - Evolution de la vitesse de fluage de la glace lors d'un essai de compression sous une contrainte constante. Fig. 2. - Creep rate versus time for ice in compression under constant load. 


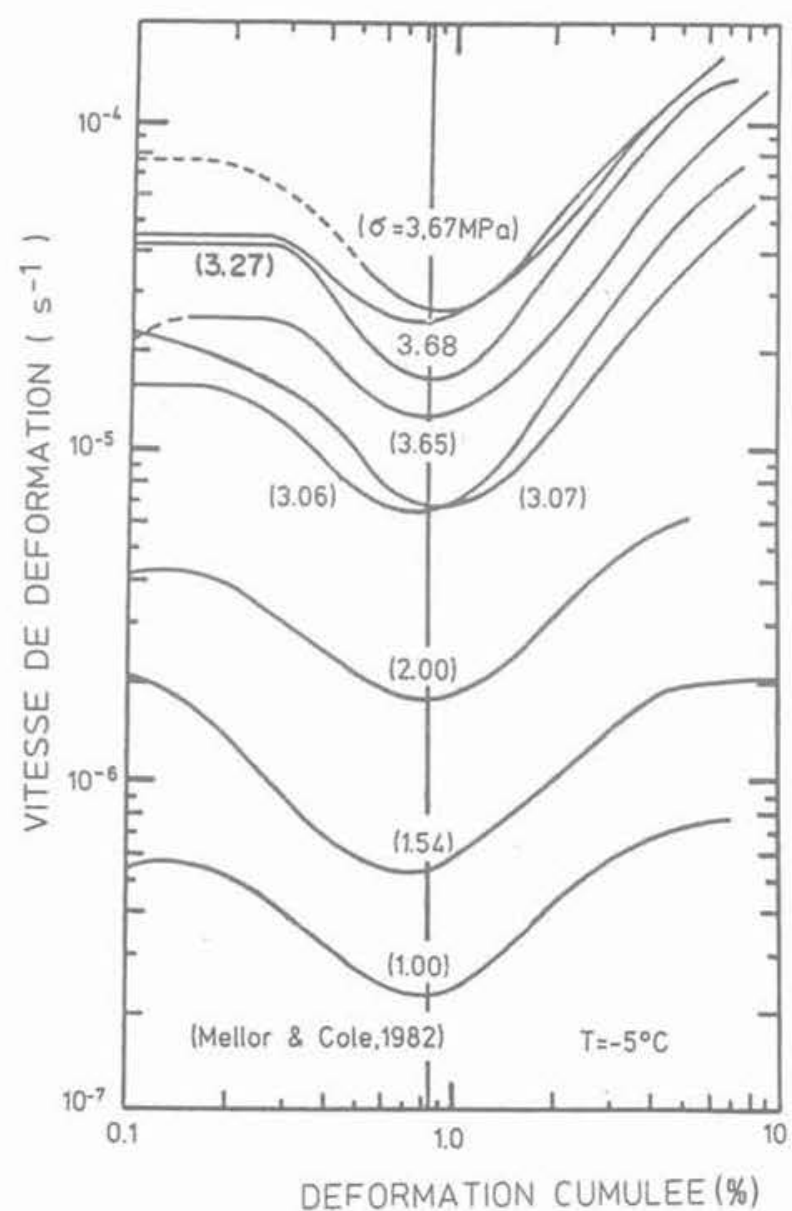

Fig. 3. - Relation entre vitesse de fluage et déformation cumulée d'une éprouvette cylindrique de glace pure.

Fig. 3. - Creep rate versus strain for ice in compression under constant load.

déduites d'essais à contrainte constante ou à vitesse de déformation constante. Par exemple, le point $\mathrm{X}$ de la figure 5 traduit une vitesse de déformation à la rupture de $2,60 \times 10^{-6} \mathrm{~s}^{-1}$ pour un essai sous une contrainte constante à $2,34 \mathrm{MPa}$; ce point $\mathrm{X}$ représente également une contrainte à la rupture de $2,32 \mathrm{MPa}$ lors d'un essai à vitesse constante de déformation égale à $2,65 \times 10^{-6} \mathrm{~s}^{-1}$.

\subsection{Rupture de la glace en compression et fluage secondaire}

En résumé, il apparaît que l'on puisse toujours atteindre la rupture de la glace "chaude "soumise à une compression axiale pourvu que les essais durent suffisamment longtemps; la rupture correspond alors invariablement à environ $1 \%$ de déformation. II apparaît également que le concept de fluage secondaire selon lequel, à contrainte constante, le fluage évolue à une vitesse constante sur une longue période de temps, soit erroné. Les figures 2 et 3 montrent en effet que la vitesse de déformation n'est jamais constante. Cependant, pour les applications courantes du génie civil, il peut être raisonnable de garder

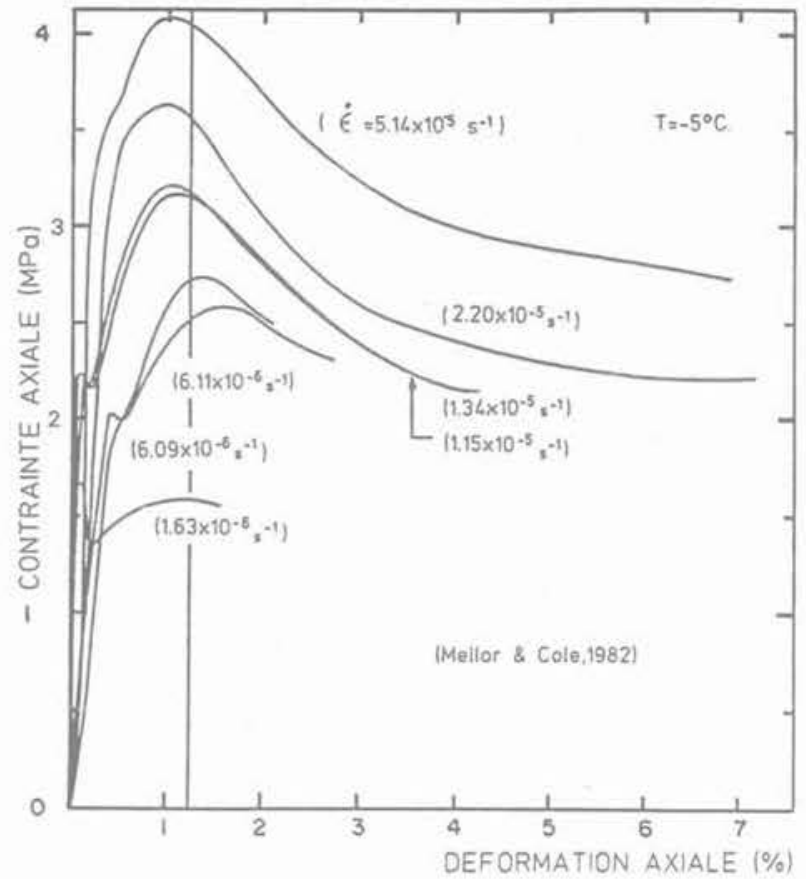

Fig. 4. - Courbes " contrainte-déformation » pour des vitesses de déformation constantes. Fig. 4. - Stress-strain response of ice in constant rate of strain tests.

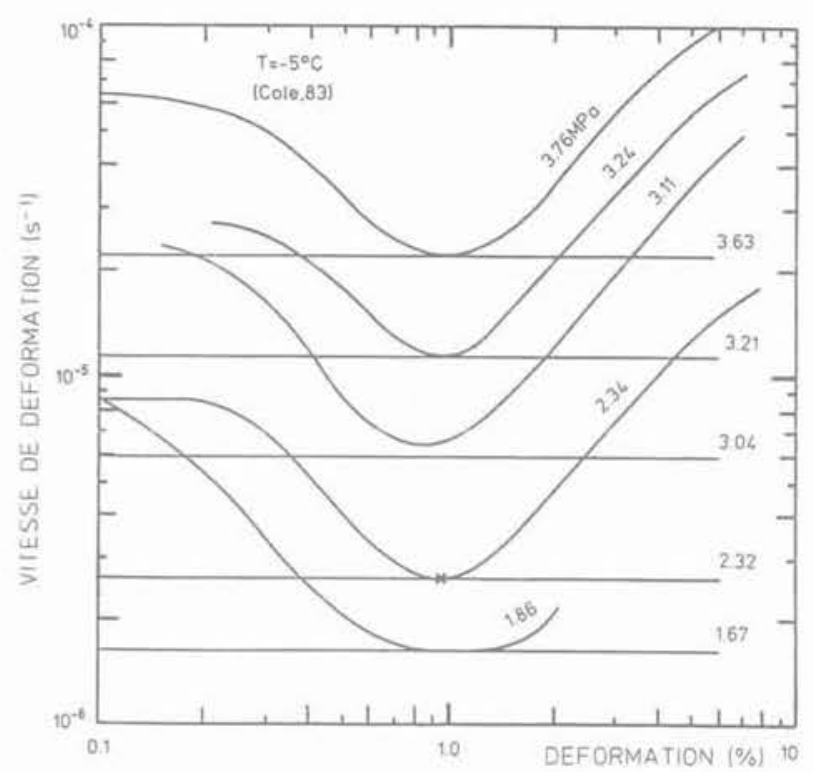

Fig. 5. - Analyse du comportement de la glace pure pour deux procédures d'essai différentes (contrainte constante et vitesse de déformation constante).

Fig. 5. - Comparison between constant rate of strain and constant stress tests of ice.

cette notion de fluage secondaire. $\mathrm{Ce}$ pseudo fluage secondaire pourrait alors être défini comme étant l'intervalle de temps ou de déformation pendant lequel la vitesse de déformation en fluage est inférieure ou égale à 1,5 fois la vitesse minimale (fig. 6). 


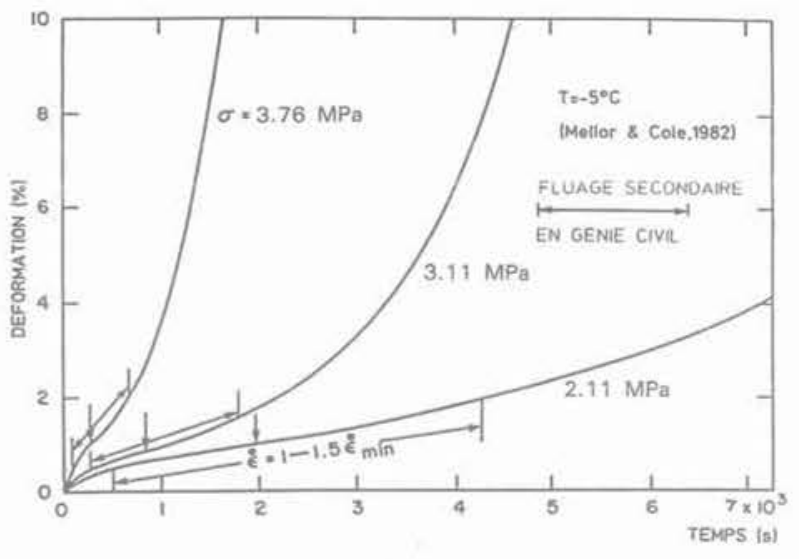

Fig. 6. - Une définition du fluage secondaire pour les applications courantes en génie civil.

Fig. 6. - Secondary creep defined for civil engineering purposes.

En génie civil, une précision de + ou - $50 \%$ peut être acceptable pour certains problèmes; c'est par exemple le cas lorsque l'on estime les vitesses de tassement de structures à partir d'essais œdométriques.

\subsection{Vitesse de déformation en fonction de la contrainte}

La figure 7 (MELLOR et COLE, 1983) montre bien que la vitesse de déformation minimum dépend en fait de la contrainte appliquée (dans le cas d'essais à contrainte constante) et que la contrainte à la rupture dépend, quant à elle, de la vitesse de déformation (dans le cas d'essais à vitesse de déformation

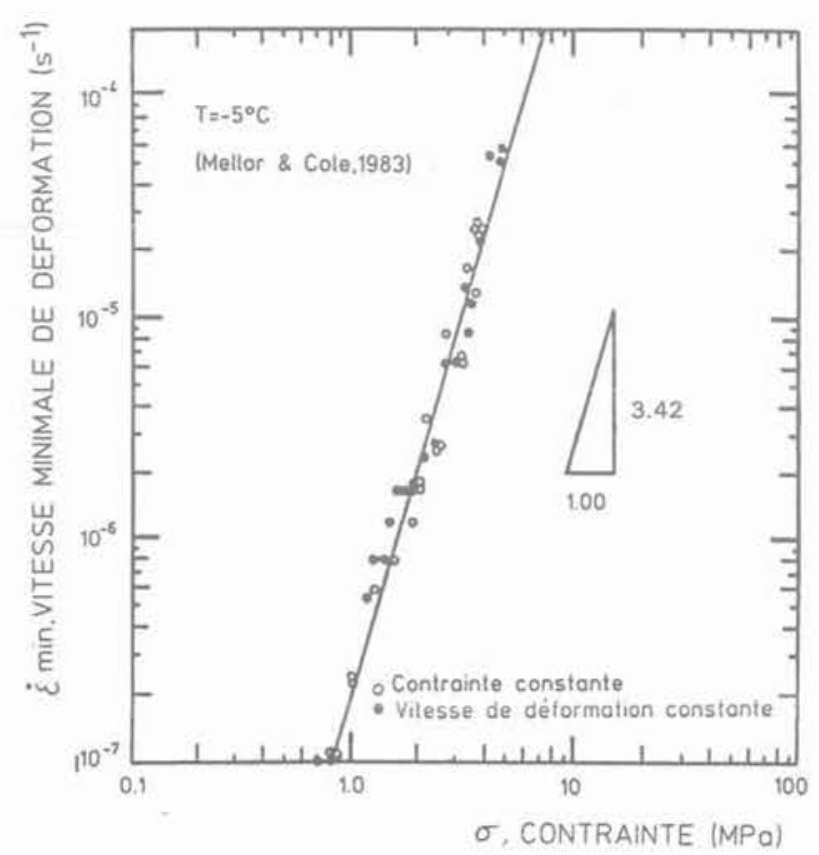

Fig. 7. - Relation entre vitesse de déformation minimale et contrainte maximale appliquée lors d'essais de compression simple sur la glace pure.

Fig. 7. - Minimum strain rate versus maximum stress for ice in compression. constante). Une fois de plus, cependant, on peut constater que la relation existant entre la contrainte à la rupture et la vitesse de déformation à la rupture est indépendante de la méthode d'essai utilisée. L'équation correspondant à la ligne droite de la figure 7 est :

$$
\dot{\epsilon}=2,14 \times 10^{-7} \sigma^{3,42} \mathrm{~s}^{-1}
$$

cette équation est de la forme :

$$
\dot{\epsilon}=\mathrm{A} \sigma^{\mathrm{n}}
$$

avec : $\dot{\epsilon}=$ vitesse de déformation axiale

$A=$ constante du matériau en fonction de la température

$\sigma=$ contrainte axiale

$\mathrm{n}=$ constante du matériau

Cette équation est souvent appellée équation de GLEN (GLEN, 1955).

On considère généralement que la loi de GLEN exprime le fait que la vitesse de déformation en fluage secondaire dépend de l'amplitude de la contrainte constante. Comme nous lavons vu cependant, cette équation exprime simplement le fait que la vitesse de déformation minimun dépend de la contrainte.

Mis à part les points correspondant à la rupture, il est également possible d'appliquer la loi de GLEN à d'autres points bien définis sur une famille de courbes contrainte-déformation. Par exemple, la contrainte à la limite élastique, pour une vitesse de déformation constante, lors d'un essai du type de celui de la figure 4 , peut être reportée sur un abaque en fonction de la vitesse de déformation imposée. (Dans Particle de MELLOR et COLE de 1982 le point de la limite élastique correspond à une déformation moyenne de $0,13 \%$ pour 22 essais ; les déformations observées étaient comprises dans l'intervalle 0,03 et $0,35 \%$ ). La figure 8 montre que les points correspondants s'alignent bien si les données sont reportées sur une échelle bilogarithmique. De la même manière, on trouve également une droite en reportant le logarithme de la contrainte pour une déformation de $10 \%$ en fonction du logarithme de la vitesse de déformation imposée (figure 9). Les valeurs de " $A$ » et $* n$ * diffèrent d'un cas à l'autre, ainsi que l'indique le tableau 1. Il est donc important de préciser la déformation à laquelle $\mathrm{A}$ et $\mathrm{n}$ sont calculés.

Tableau 1. - Les paramètres de la loi de Glen en fonction de la déformation

\begin{tabular}{|c|c|c|}
\hline$\epsilon$ & ${ }^{\mathrm{A}}$ & $n$ \\
\hline Limite élastique & $3,26 \times 10^{-7}$ & 3,94 \\
\hline$\dot{\epsilon}_{\min }$ & $2,14 \times 10^{-7}$ & 3,42 \\
\hline $10 \%$ & $8,93 \times 10^{-7}$ & 4,03 \\
\hline
\end{tabular}




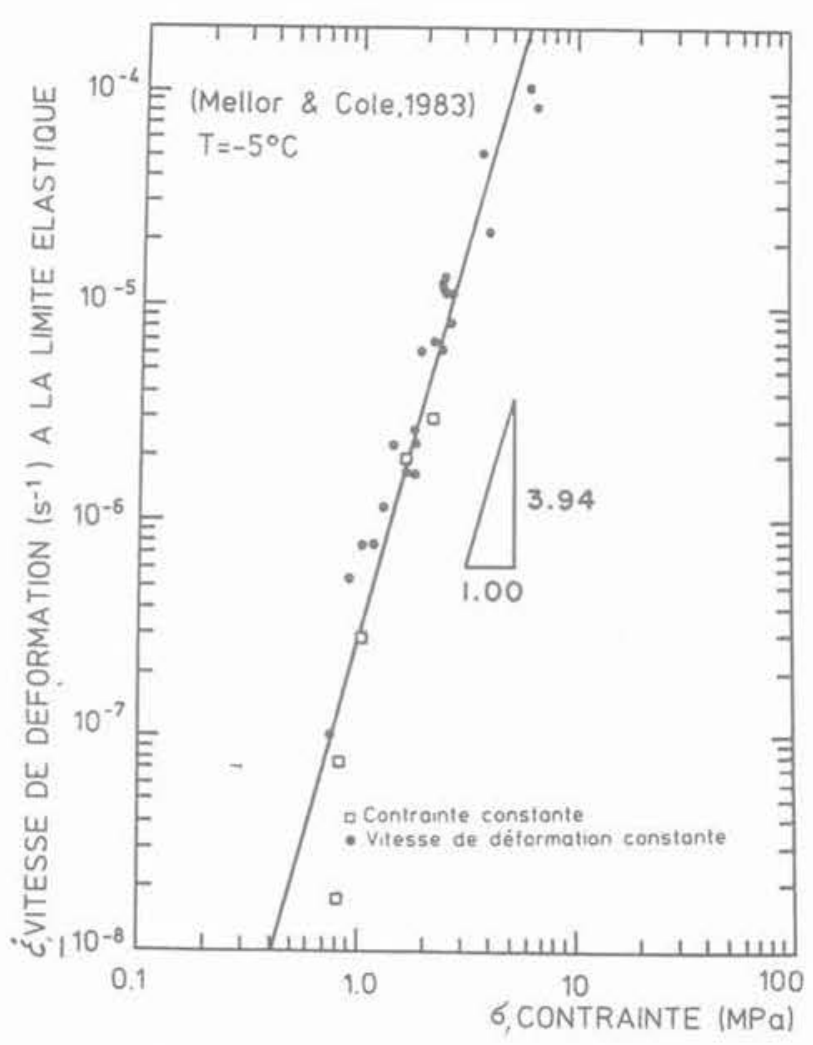

Fig. 8. - Relation entre vitesse de déformation et contrainte appliquée à la limite élastique de la glace pure.

Fig. 8. - Stress versus strain rate at initial yield of ice in compression.

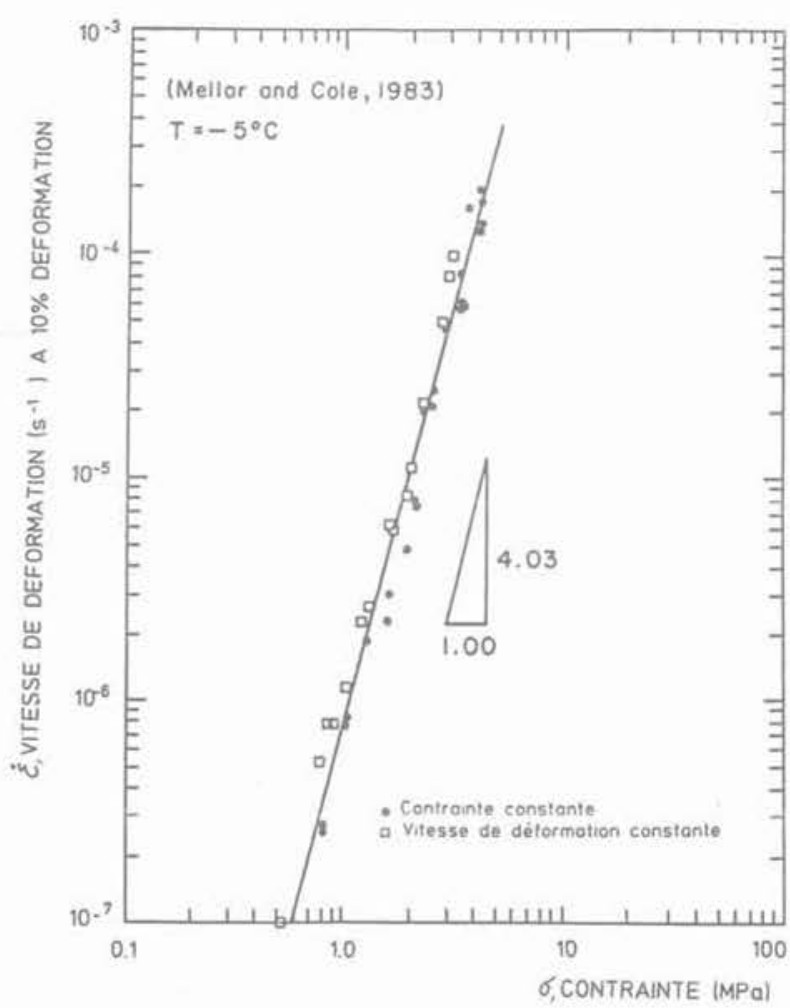

Fig. 9. - Relation entre vitesse de déformation et contrainte appliquée à $10 \%$ de déformation.

Fig. 9. - Stress versus strain rate at 10 per cent strain for ice in compression.
La figure 10 (JACKA, 1984) traduit quant à elle la complexité des phénomènes observés dans le domaine des faibles contraintes. JACKA a aussi réalisé des essais de compression à contrainte constante pour des niveaux de contrainte aussi faibles que 0,1 $\mathrm{MPa}$ (sous ce niveau de contrainte, il a fallu 3 ans pour atteindre la vitesse minimum de déformation). Il ne fait aucun doute qu'on obtiendrait le même résultat pour une vitesse constante de déformation très faible. Pour toutes les températures considérées, on observe, dans le domaine des faibles contraintes, que les points expérimentaux s'écartent de la droite correspondant à la loi de GLEN dans un diagramme bilogarithmique. JACKA en conclut que \& la meilleure manière d'établir une relatlion empirique entre la contrainte et la vitesse de déformation minimum est d'utiliser une fonction sh plutôt que d'appliquer une loi de puissance ». Cette allure avait déjà été observée précédemment par MELLOR en 1979.

On retiendra qu'il convient de travailler en laboratoire sous un niveau de contrainte relativement élevé si l'on veut à la fois effectuer l'essai dans un intervalle de temps raisonnable et, également, assurer une préci-

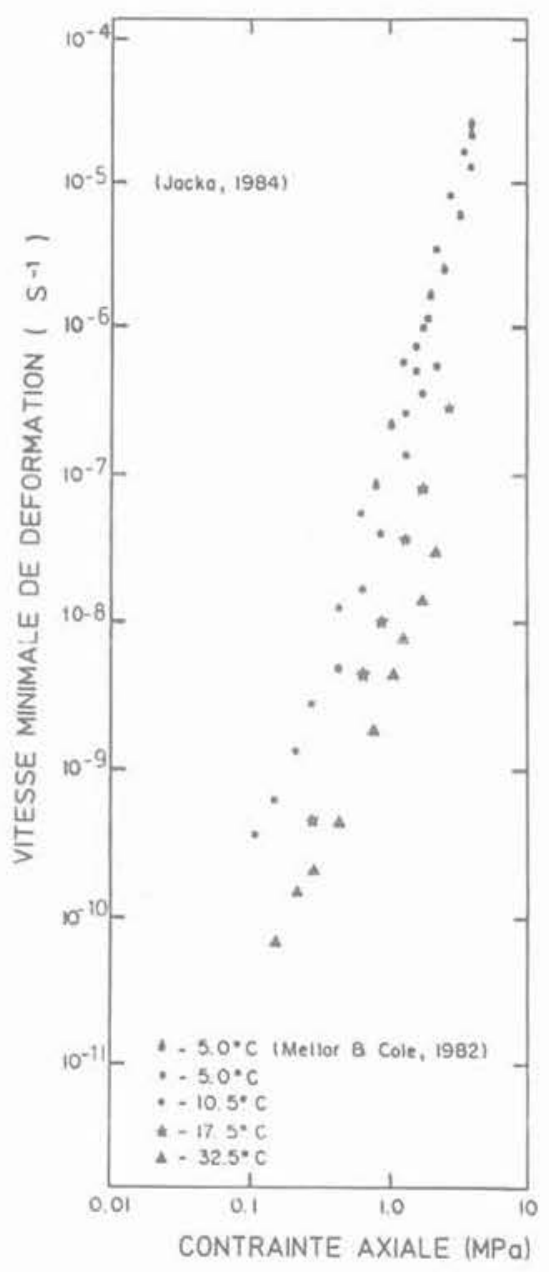

Fig. 10. - Non-linéarité de la courbe

"vitesse minimale - contrainte appliquée ":

Cas du domaine des faibles contraintes.

Fig. 10. - The result of extending the stress range for compression tests on ice. 
sion suffisante aux mesures. La contrainte transmise par une fondation, in situ, excèdent rarement $0,1 \mathrm{MPa}$. Dans les faits, les courbes de la figure 10 n'en sont, par contre, que plus intéressantes pour une application pratique en génie civil.

\subsection{Influence de la température}

La figure 10 illustre également linfluence de la température sur le fluage de la glace. Si l'on raisonne sur les constantes $A$ et $n$ de la loi de GLEN, il ressort clairement que le facteur $\approx A$ » est nettement plus sensible à la température que l'exposant « $n$ ».

L'influence de la température sur la vitesse du fluage de la glace peut être prise en compte (SEGO, 1980) par lexpression relativement simple proposée par VOYTKOVSKIY (1960) :

$$
\dot{\epsilon}=f\left(\frac{1}{1-T}\right)
$$

où : $\mathrm{T}=$ température en ${ }^{\circ} \mathrm{C}$ pour une plage de températures comprises entre 0 et $-10^{\circ} \mathrm{C}$.

Les données expérimentales, correspondant à des températures inférieures à $-10^{\circ} \mathrm{C}$, fournies par MELLOR (1979) sont, quant à elles, compatibles avec l'équation d'Arrhénius :

$$
\dot{\epsilon}=f(\exp (g T))
$$

où : $\mathrm{g}=$ une constante.

JACKA semble avoir été le premier à prouver, lors d'essais de compression à charge constante, que la relation entre la vitesse de déformation minimale et le temps nécessaire pour l'atteindre (c'est-à-dire le temps écoulé jusqu'à la rupture) est indépendant de la température (fig. 11). Tous les résultats indiqués sont en effet regroupés sur une courbe unique. Cette caractéristique peut être partiellement attribuée au fait que la déformation à la rupture soit constamment égale à $1 \%$.

Ce résultat plus ou moins approché dans le cas cidessus est, par contre, aisément démontrable dans le cas des essais à vitesse de déformation constante. En effet, le temps nécessaire pour atteindre la rupture est, dans ce type d'essai, directement proportionnel à la vitesse de déformation imposée, elle-même par nature indépendante de la température. Il s'ensuit donc forcément que le temps soit lui aussi indépendant de ce facteur.

\subsection{Fluage primaire}

Un certain nombre de chercheurs parmi lesquels GARDNER, JONES et HARRIS (1984) ; ASHBY et DUVAL (1985); SZYSZKOWSKI et GLOCKNER (1985) ont essayé de décrire la première partie des courbes de fluage de la figure 1. L'approche la plus simple est probablement de supposer que le fluage primaire obéit à l'équation :

$$
\epsilon=B \cdot \mathrm{t}^{\mathrm{b}}
$$

où : $\epsilon=$ déformation par fluage

$\mathrm{B}=$ une constante pour un matériau, une contrainte et une température données

$\mathrm{t}=\mathrm{le}$ temps

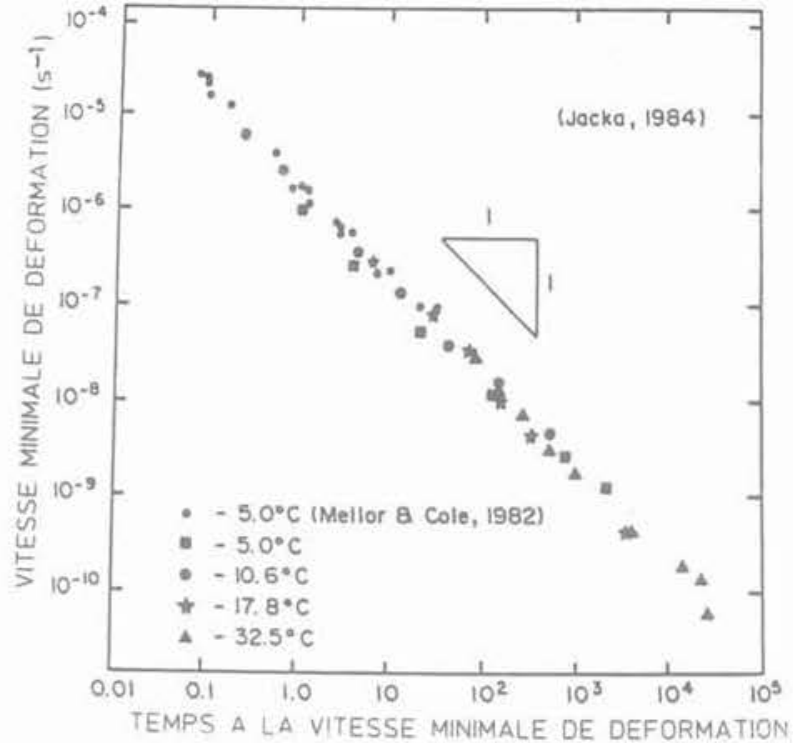

(h)

Fig. 11. - Analyse d'essais de compression simple sur la glace : relation entre vitesse de déformation minimale et temps nécessaire pour atteindre la rupture.

Fig. 11. - Time to failure for compression tests on ice.

$b=$ une constante possédant une valeur inférieure à l'unité et dépendant du matériau, de la contrainte et de la température.

Cette équation implique que les résultats d'essais devraient être linéarisables sur une courbe $\log \epsilon$ en fonction de $\log t$, du moins pour des déformations inférieures ou égales à la déformation à la rupture $\epsilon_{\mathrm{f}}$. La figure 12 regroupe les résultats d'essais de compression de MELLOR et COLE, reportés suivant cette

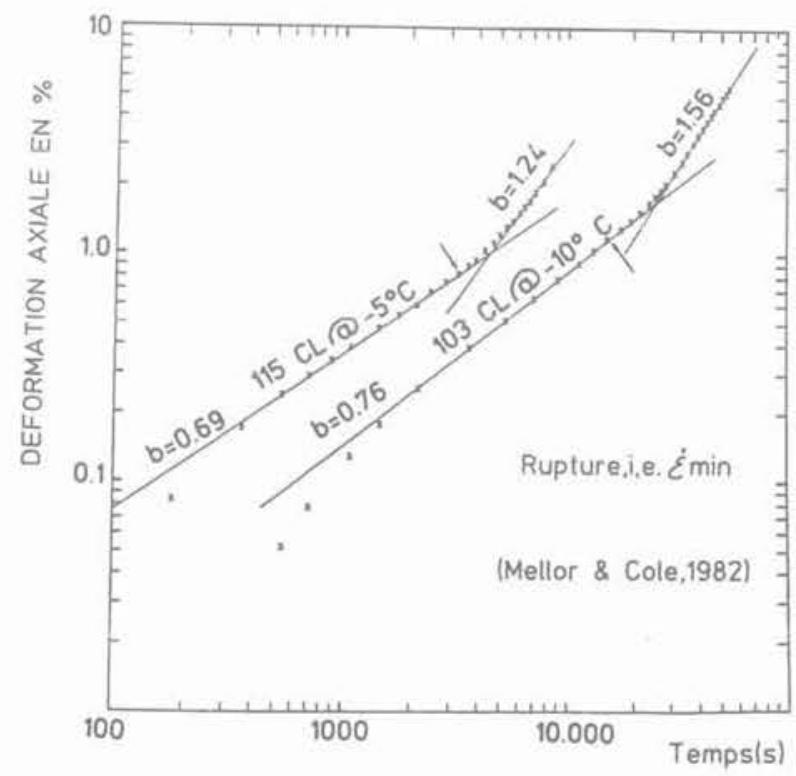

Fig. 12. - Analyse d'essais de compression simple sur la glace: application d'une loi "puissance ” aux courbes « contraintes-déformations $n$.

Fig. 12. - Fitting a power law to primary creep in a constant stress compression test. 
représentation. A l'exception de deux ou trois points en début d'essai, un calage linéaire semble raisonnable sur toute la courbe jusqu'à la rupture (indiquée par la flèche). (Il est intéressant de noter qu'une ligne droite peut être aussi ajustée sur les données correspondant à des déformations supérieures à $\epsilon_{i}$, après une période de transition; cette seconde ligne droite peut être également décrite par une équation de la même forme :

$$
\epsilon=C \cdot t^{b}
$$

où : $\mathrm{C}=$ une constante

$\mathrm{b}=$ une constante supérieure à l'unité).

La figure 12 semblerait prouver qu'il ne soit pas, dans le cas des essais de laboratoire, nécessaire de recourir à des formulations plus complexes du fluage primaire : on verra cependant ci-après qu'il n'en sera pas de même dans le cas des essais pressiométriques.

\section{ESSAIS AU PRESSIOMÈTRE}

\subsection{Equipement et procédures d'essai}

Les essais pressiométriques ont été réalisés dans 8 éprouvettes identiques de glace polycristalline artificielle, formée à partir d'eau douce (KJARTANSON, 1986). Ces éprouvettes cylindriques, de $890 \mathrm{~mm}$ de diamètre et $800 \mathrm{~mm}$ de hauteur, sont installées à P'intérieur d'une cuve d'acier (fig. 13). Un pressiomètre électronique monocellulaire OYO, de $70 \mathrm{~mm}$ de diamètre, a été placé par la suite dans un avant-trou creusé dans laxe des blocs de glace et sur toute leur hauteur, avec une tarière de type « CRREL ».

La sonde pressiométrique a été ensuite gonflée, en un seul palier, jusqu'à la pression retenue pour chacun des essais, dont le plus long a duré 50 jours. Dans la pratique, chaque essai a été poursuivi jusqu'au doublement du volume initial de la sonde. Dans tous les cas, un système de réfrigération a permis de maintenir constante la température de la glace à la valeur de $-2,0 \pm 0,2^{\circ} \mathrm{C}$.

\subsection{Relation « vitesse de déformation-temps * et « vitesse de déformation-déformation »}

La figure 14 représente l'évolution du rayon de la cavité centrale en fonction du temps pour 7 des 8 essais pressiométriques à contrainte constante. Ces courbes ont subi des corrections intégrant à la fois la résistance et l'épaisseur de la membrane en caoutchouc du pressiomètre. La figure 15 présente les mêmes résultats d'essais reportés dans les axes log $(\dot{r} / r)$ en fonction de $\log t$, où :

$r=$ rayon de la cavité au temps $t$,

et $\hat{r} / r=$ la vitesse d'allongement de la circonférence de la cavité, considérée comme «vitesse de déformation ».

(Notons, au passage, que sur cette figure 15 apparaissent les résultats des 8 essais au pressiomètre formant le corps de cet article ; 2 des 8 essais au pressiomètre ont été effectués sous une pression de 2,0 $\mathrm{MPa}$ afin de vérifier la répétabilité des résultats). De

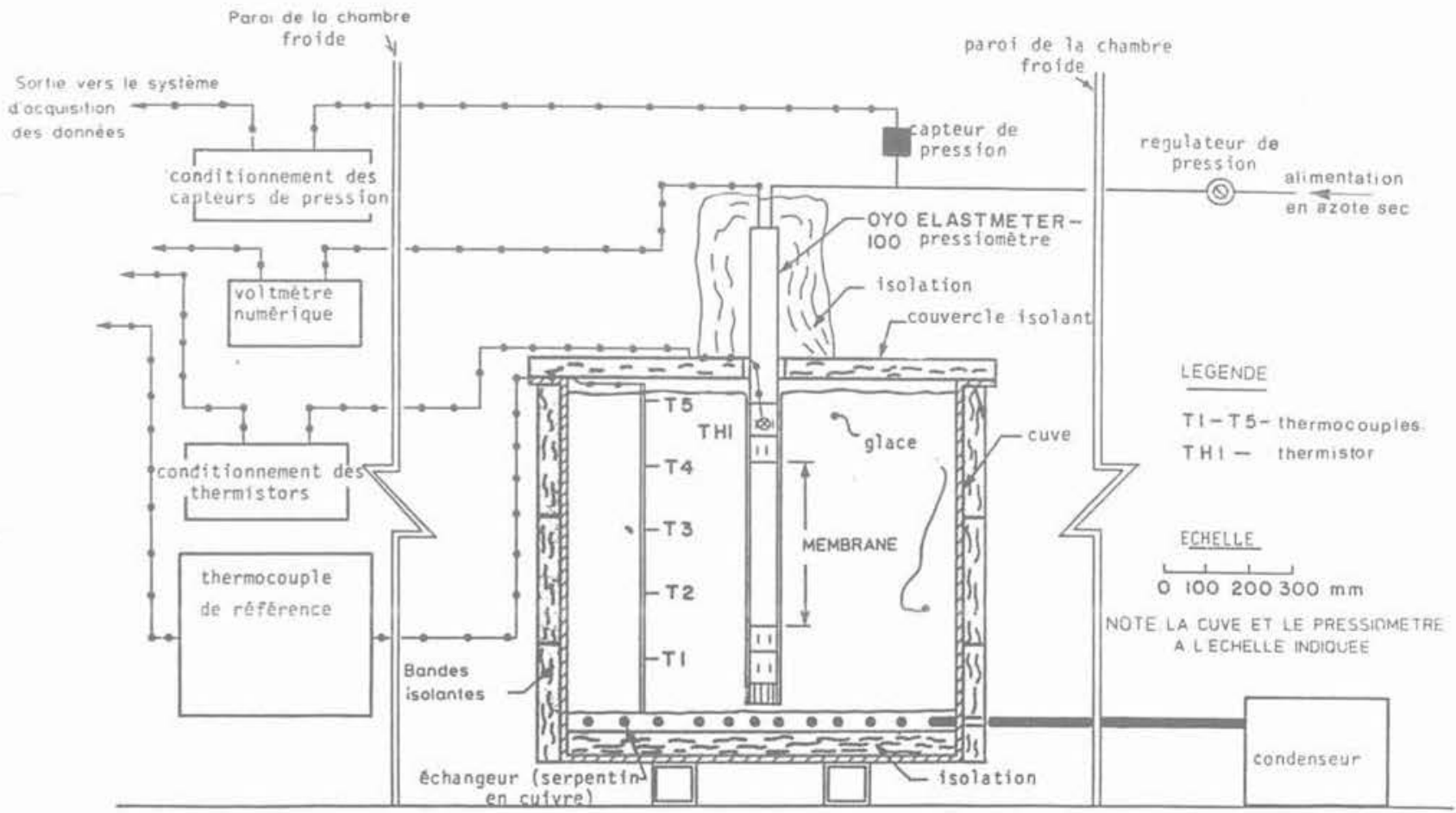

Fig, 13. - Principe de réalisation d'essais pressiométriques en laboratoire. Fig. 13. - Schematic of pressuremeter test set-up. 


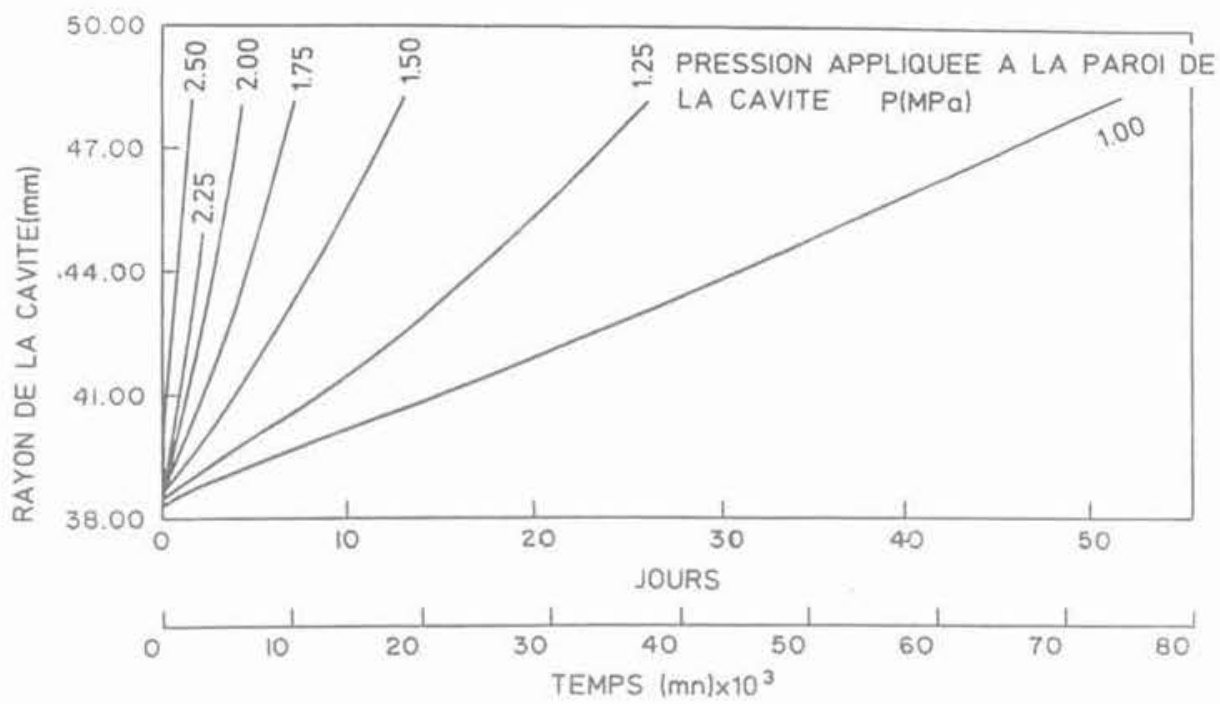

Fig. 14. - Fluage de la glace lors d'un essai pressiométrique à pression constante. Fig. 14. - Creep of ice measured in a pressuremeter test at constant pressure.

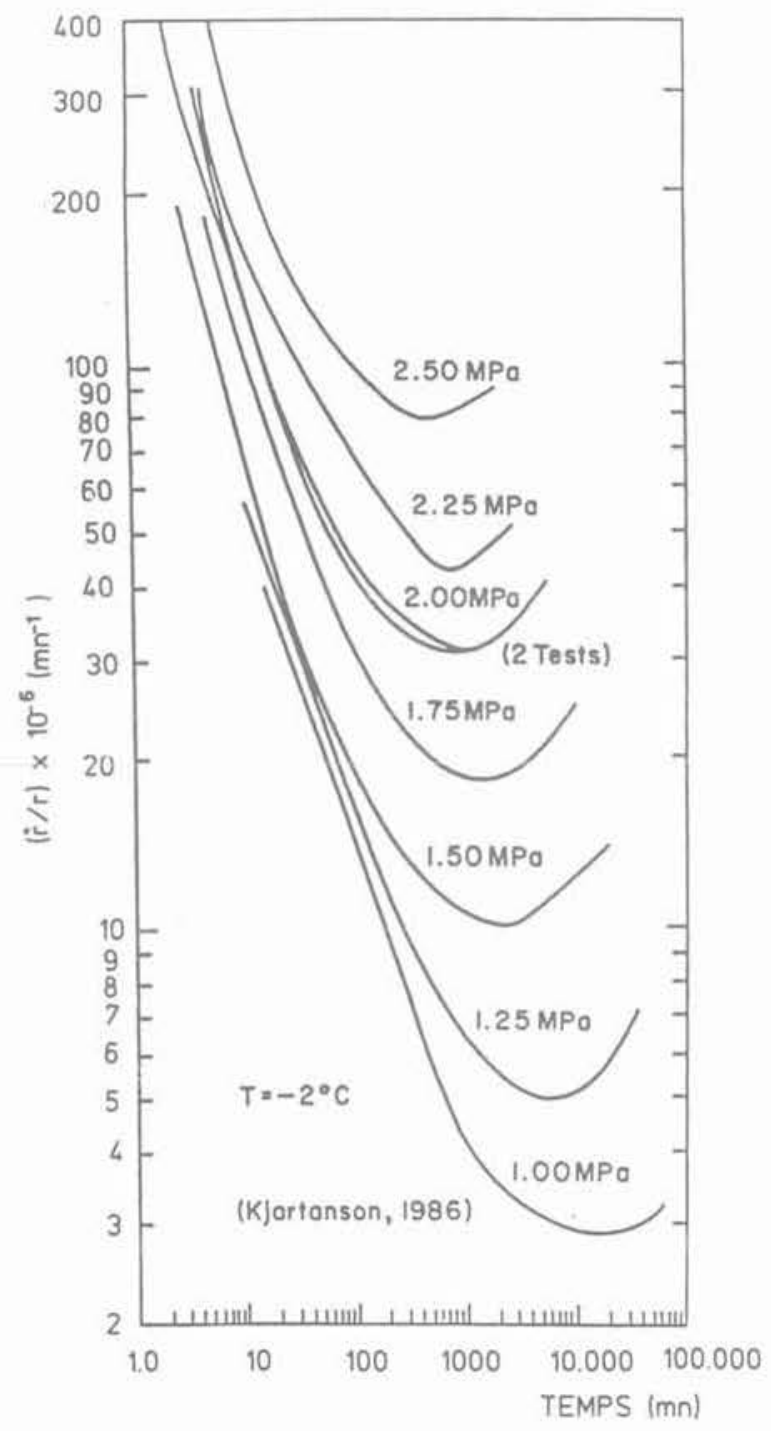

Fig. 15. - Evolution de la vitesse de déformation en fonction du temps lors des essais pressiométriques dans la glace pure.

Fig. 15. - Strain rate versus time for pressuremeter test at constant pressure in ice. même que pour les essais de compression, on trouve une vitesse de déformation minimum clairement définie pour chaque essai au pressiomètre; la vitesse de déformation minimum apparait à un moment précis et, une fois de plus, il n'y a pas fluage secondaire.

Lorsque l'on représente le logarithme de la vitesse de déformation en fonction du logarithme de la déformation (fig. 16), c'est-à-dire $\log (\dot{r} / \mathrm{r})$ en fonction de $\log \left(\Delta r / r_{0}\right)$ (avec $r_{0}=$ rayon initial du forage et $\left.\Delta r=r-r_{0}\right)$, la rupture est atteinte, lors des essais au pressiomètre, pour une déformation comprise entre 3 et $4 \%$ au lieu de $1 \%$ comme cela fut le cas lors des essais de compression (fig. 3). On peut expliquer la différence existant entre la déformation à la rupture lors des essais au pressiomètre et lors des essais de compression de la manière suivante:

- la glace se trouvant autour du pressiomètre est soumise à un cisaillement pur lors duquel la diminution de la longueur d'un élément sur un rayon (fig. 17) est égale, au moins au début de l'essai, à laugmentation de sa largeur. Par ailleurs, lors d'un essai de compression, la contrainte latérale reste constante (généralement nulle), tandis que la contrainte verticale augmente. Les chemins de contrainte représentatifs des deux types d'essais sont donc bien différents (voir fig. 18);

- il se produit une redistribution des contraintes au sein du tube épais de glace. Initialement, celle-ci aura un comportement élastique avec une distribution donnée de contrainte dans le tube (voir fig. 19), qui évoluera dans le temps au fur et à mesure de lapparition du fluage du matériau. Au bout d'un certain temps, cette distribution des contraintes se stabilisera (du moins théoriquement) et l'état de fluage stationnaire sera atteint. Le champ de variation des contraintes doit avoir une influence sur le processus de fluage. Une telle redistribution des contraintes ne se produit pas par contre à l'intérieur d'un cylindre de glace soumis à l'essai de compression simple. 


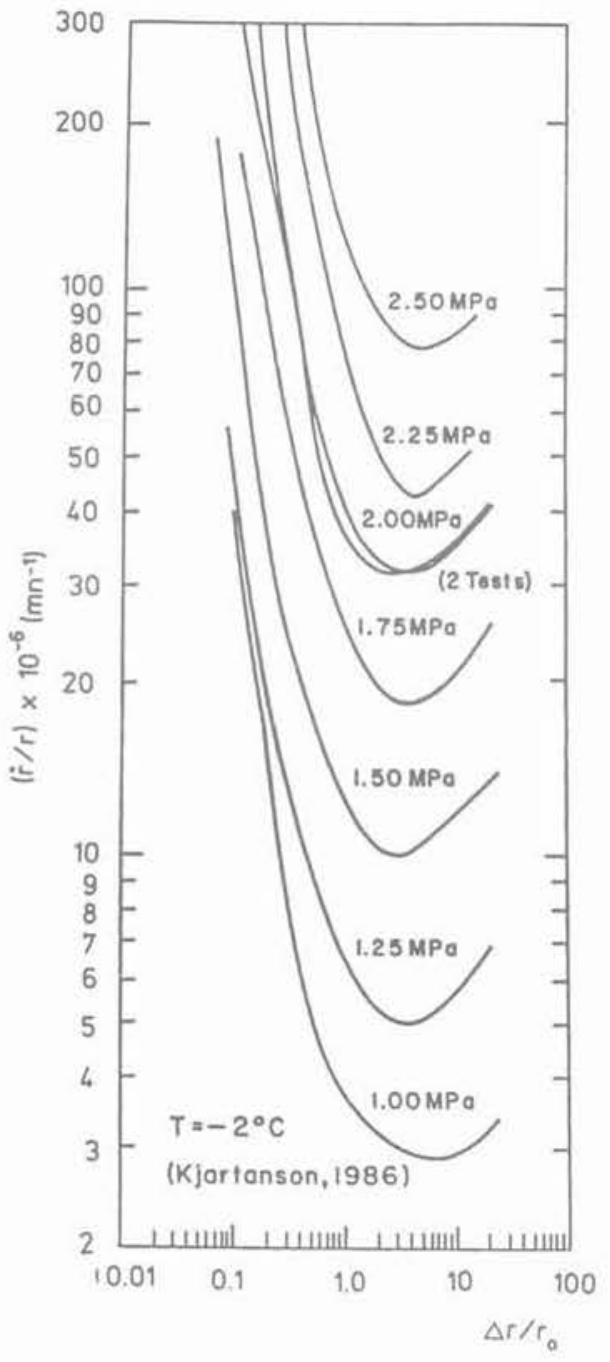

Fig. 16. - Evolution de la vitesse de déformation en fonction de la déformation mesurée lors des essais pressiométriques dans la glace pure.

Fig. 16, - Strain rate versus strain for pressuremeter test at constant pressure in ice.

\subsection{Relation}

\section{«vitesse de déformation-contrainte »}

La loi de l'écoulement de GLEN, généralisée à des états multiaxiaux de contraintes par NYE en 1957. suppose que la glace polycristalline se comporte comme un fluide incompressible (loi puissance) dont l'équation peut être écrite de la manière suivante:

$$
\mathrm{T}+\mathrm{PI}=2 \eta \pi^{\mathrm{m} / 2} \mathrm{D}
$$

où : T est le tenseur de contrainte de Cauchy ;

$\mathrm{P}$ est la pression isotrope (indéterminée) due

à l'incompressibilité ;

I est le tenseur identité ;

$\mathrm{D}$ est le tenseur vitesse de déformation;

$\pi=1 / 2\left(\right.$ trace $\left.D^{2}\right)$;

$\eta$ et $\mathrm{m}$ sont des coefficients du matériau qui dépendent généralement de la température.

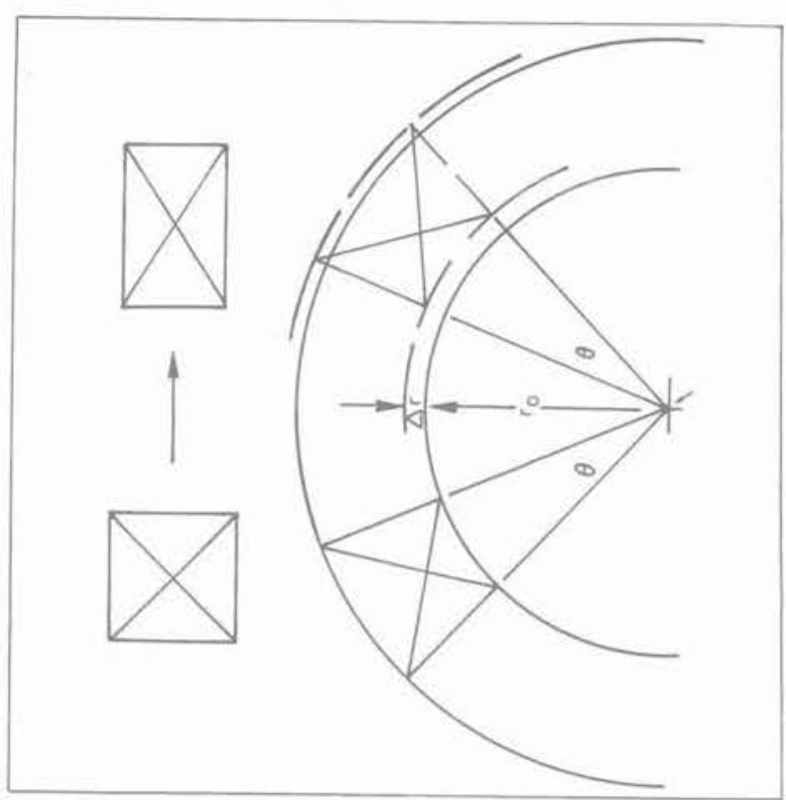

Fig. 17. - Schématisation du cisaillement de la glace autour du pressiomètre.

Fig. 17. - Shear deformation around a pressuremeter.

En ce qui concerne les essais de compression triaxiale (et uniaxiale), dans l'hypothèse où l'équation 7 peut s'appliquer lorsque la vitesse de déformation axiale est minimale, on retrouve alors l'équation de fluage de GLEN :

$$
\dot{\epsilon}_{\min }=A \sigma^{\mathrm{n}}
$$

où $\mathrm{n}$ et $\mathrm{A}$ sont reliés aux coefficients du matériau $\mathrm{m}$ et $\eta$ par l'intermédiaire des équations (9) et (10) suivantes :

$$
\mathrm{n}=1 /(\mathrm{m}+1)
$$

$$
A=2^{1-n} 3^{-(1+n) / 2} \eta^{-n}
$$

Dans le cas des essais au pressiomètre, la pression $\mathrm{p}_{\mathrm{o}}$ s'exerçant loin du forage est généralement inconnue, l'hypothèse $\mathrm{p}_{0}=0$ permet cependant de déduire de l'équation 7 l'équation de fluage suivante:

$$
(\dot{r} / \mathrm{r})_{\min }=\operatorname{ap}^{\mathrm{n}}
$$

où :

$$
\mathrm{a}=(2 \mathrm{n})^{-\mathrm{n}} \eta^{-\mathrm{n}}
$$

dans lesquelles $r$ désigne le rayon du forage, $(\dot{r} / \mathrm{r})_{\min }$ la vitesse de déformation minimale de sa circonférence et $\mathrm{p}$ la pression appliquée latéralement sur le forage. Les exposants des équations 8 et 11 sont identiques ; les coefficients $\mathrm{A}$ et a peuvent être reliés par l'équation :

$$
A=2(3)^{-(1+n) / 2} n^{n} a
$$




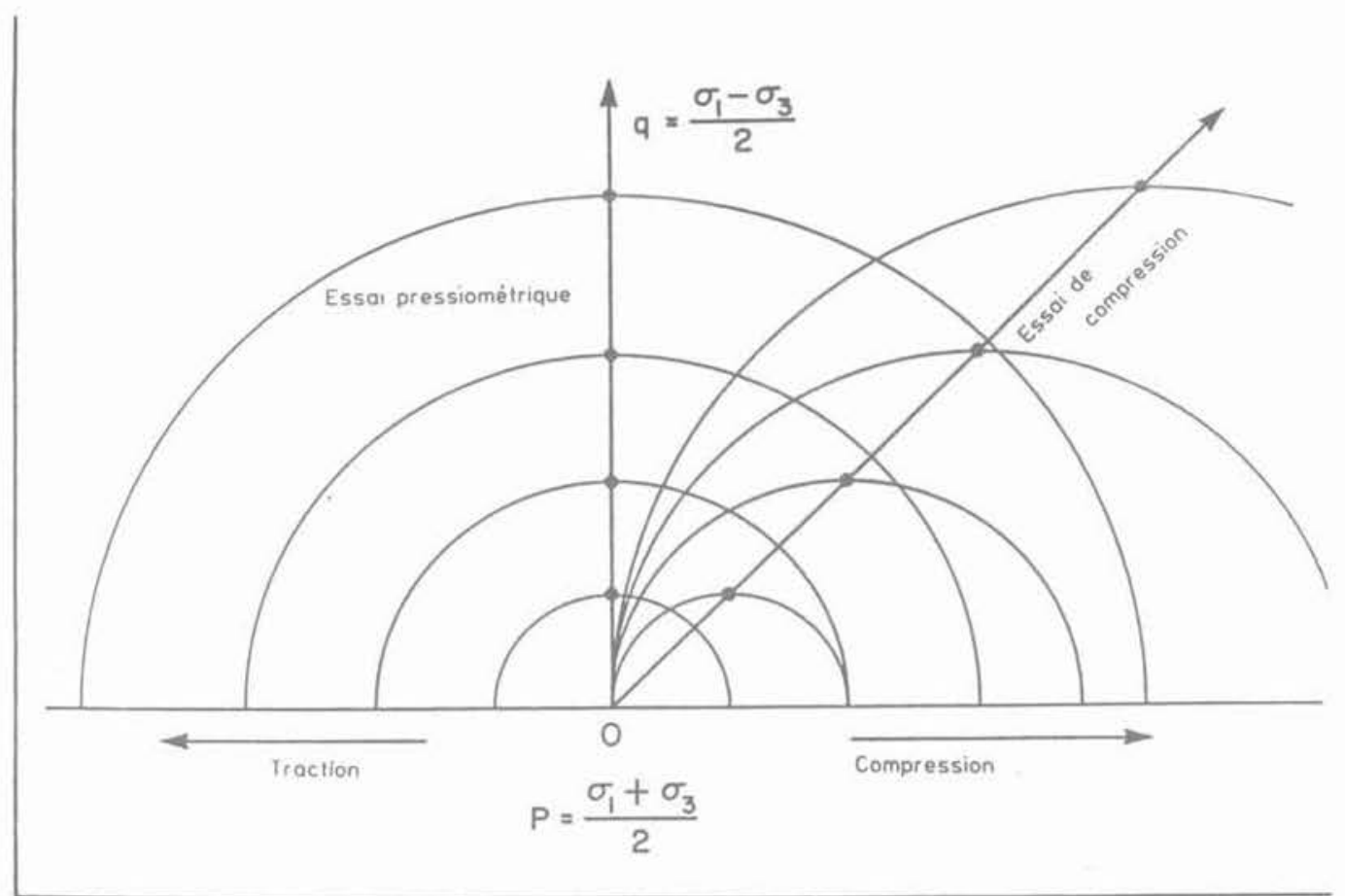

Fig. 18. - Chemin de contrainte d'un essai de compression et d'un essai pressiométrique. Fig. 18. - Stress paths for pressuremeter and compression tests.

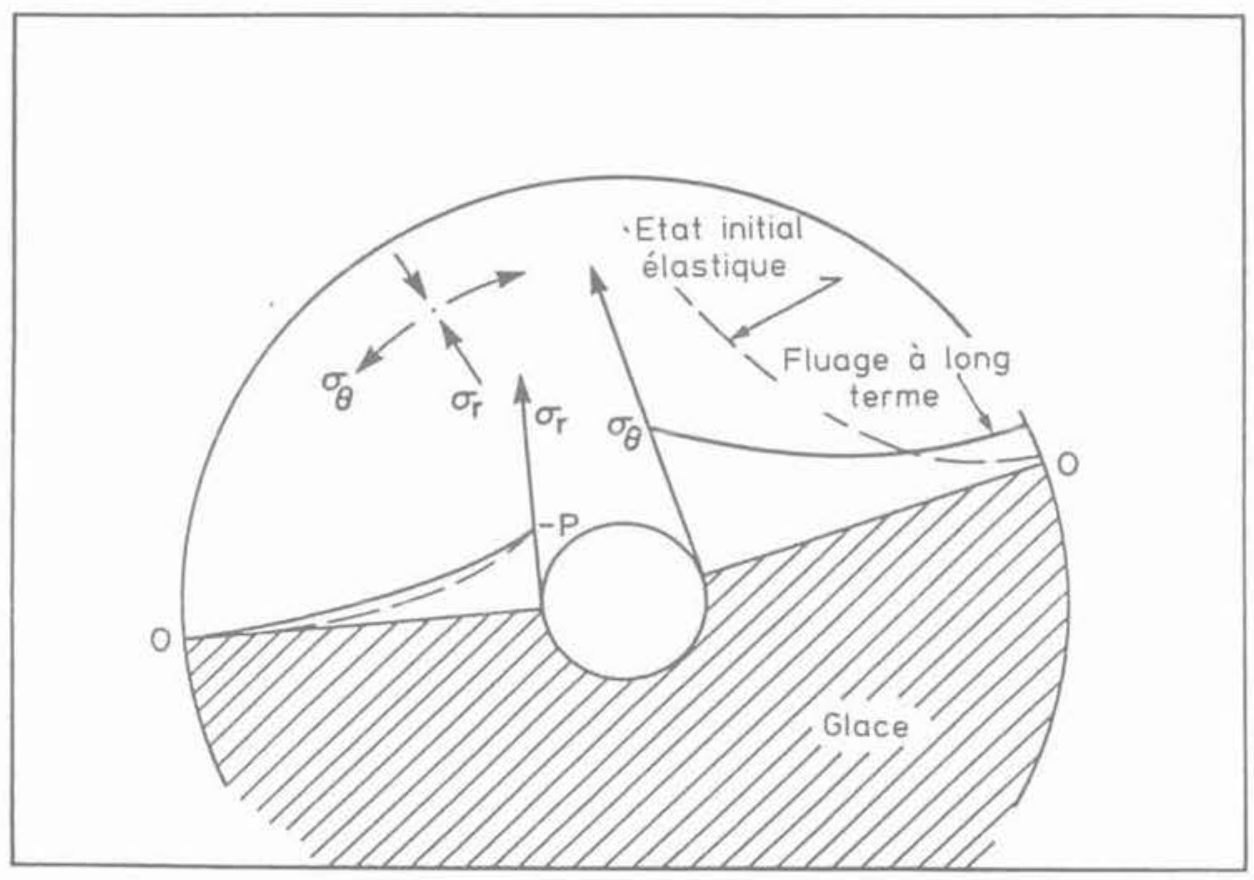

Fig. 19. - Evolution dans le temps de la répartition des contraintes au sein d'un cylindre épais de matériau viscoélastique. Fig. 19. - Stress redistribution in a thick cylinder of visco-elastic material over time.

Sur la figure 20 est représenté le logarithme de $(\dot{r} / r)_{\min }$ pour les 8 essais pressiométriques, en fonction du logarithme de $\mathrm{p}$. La pente de la droite de régression devrait donner la valeur de l'exposant $n$ s'appliquant à la loi de GLEN. Pour cette glace polycristalline, à une température de $-2{ }^{\circ} \mathrm{C}$, cette valeur est de 3,76, du même ordre de grandeur que les valeurs de n trouvées par MELLOR et COLE et par JACKA pour des contraintes sensiblement équivalentes.

Il est également possible d'estimer le paramètre a de l'équation 11 à partir des résultats déduits par des essais au pressiomètre (fig. 20). Si l'on introduit les 


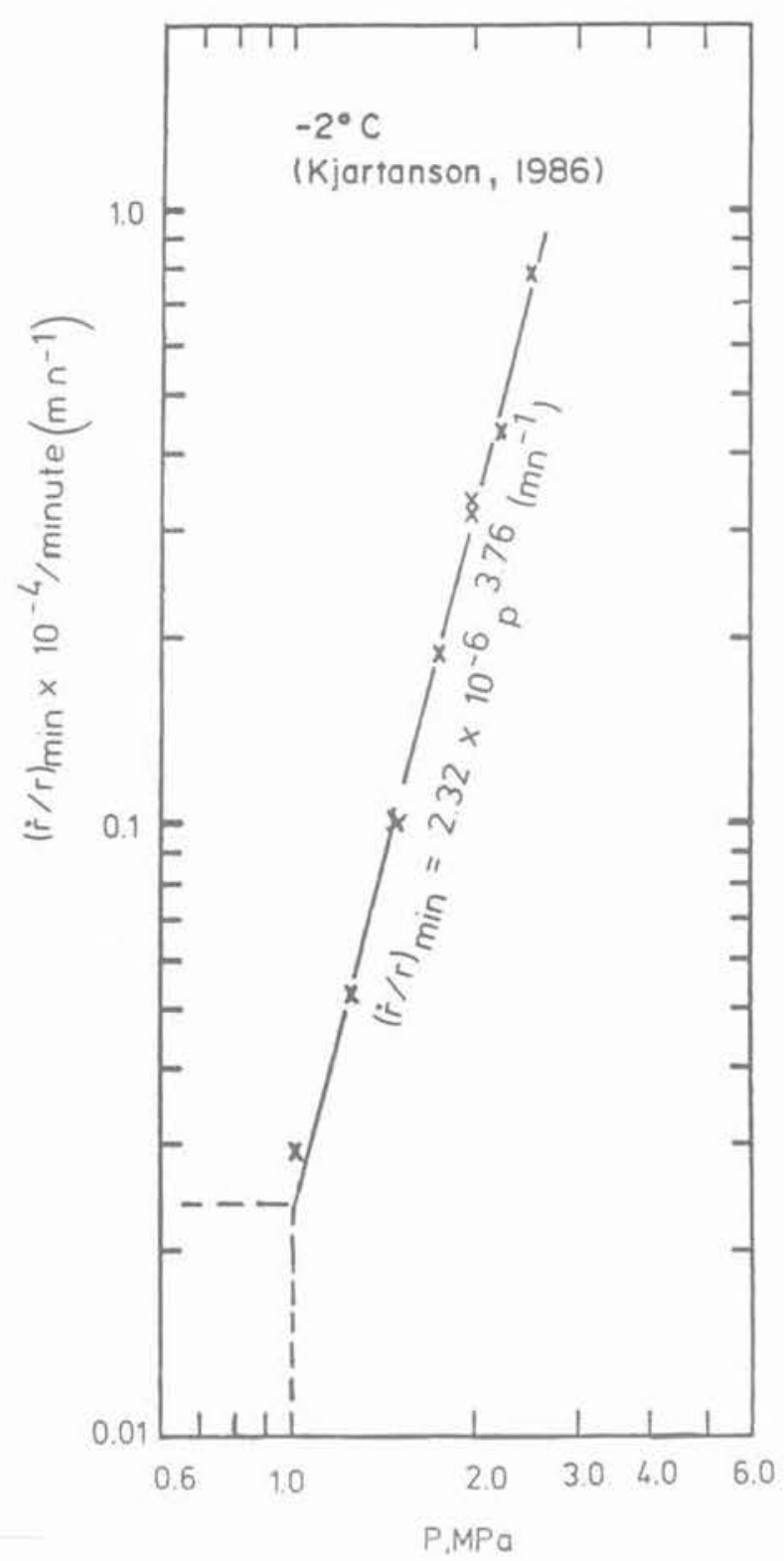

Fig. 20. - Relation « vitesse de déformation minimale - pression appliquée

lors d'une série d'essais pressiométriques à pression constante.

Fig. 20. - Pressure versus minimum strain rate

for constant pressure pressuremeter tests in ice.

valeurs ainsi déduites de a et $\mathrm{n}$ dans l'équation 13 , on obtiendra alors la valeur A telle que fournie par nos essais pressiométriques. Il est intéressant, à ce stade, d'établir une comparaison entre la valeur de A au pressiomètre et celle déduite des résultats expérimentaux de MELLOR et COLE. Une comparaison de ce type peut être effectuée en recalculant les résultats de MELLOR et COLE de la figure 7 ( $\mathrm{T}=-$ $5^{\circ} \mathrm{C}$ ) pour une température de $-2^{\circ} \mathrm{C}$ par le biais de l'équation de Voytkovskiy. On obtient alors :

$$
\dot{\epsilon}_{\min }=4,3 \times 10^{-7} \sigma^{3,42} \mathrm{~s}^{-1}
$$

(résultat obtenu par les essais de compression uniaxiale)

$$
\begin{gathered}
\dot{\epsilon}_{\min }=8,2 \times 10^{-7} \sigma^{3,76} \mathrm{~s}^{-1} \\
\text { (résultat prédit au moyen } \\
\text { des essais pressiométriques) }
\end{gathered}
$$

La concordance est, là aussi, tout à fait satisfaisante.

Les valeurs de $\mathrm{n}$ et $\mathrm{A}$ ainsi calculées dépendent:

- des valeurs de $(\dot{r} / r)_{\min }$ telles qu'elles ont été estimées à partir des données brutes du rayon $r$ en fonction du temps $\mathrm{t}$;

- du choix du lissage optimal des données.

Les valeurs estimées à partir de la méthode des moindres carrés et par une régression linéaire sur le logarithme $(\dot{r} / r)_{\min }$ en fonction du logarithme de $\mathrm{p}$ seront généralement différentes de celles obtenues par la méthode de moindres carrés mais par une régression non linéaire sur les valeurs de $(\dot{r} / r)_{\min }$ en fonction de p. Les valeurs de $n=3,76$ et $a=2,32 \times 10^{-6}$ $(\mathrm{MPa})^{-n} \min ^{-1}$ (donc $\mathrm{A}=8,2 \times 10^{-7}(\mathrm{MPa})^{-n} \mathrm{~s}^{-1}$ ) ont été obtenues à partir d'une régression linéaire sur les logarithmes. SUN (1987) a procédé à une réestimation des valeurs de $\mathrm{n}$ et $\eta$ en utilisant une régression non linéaire. Il utilise pour réaliser son lissage les données déduites des essais de fluage à paliers multiples de KJARTANSON (1986) et il utilise sa propre estimation de $(\dot{r} / r)_{\text {min }}$ pour les essais à un seul palier, décrits précédemment. Sur la base des estimations de SUN, l'équation de fluage déduite du pressiomètre, mais applicable aux essais de compression uniaxiale et triaxiale, serait :

$$
\dot{\epsilon}_{\min }=6,9 \times 10^{-7} \sigma^{3,46} \mathrm{~s}^{-1} ;
$$

cette équation se rapproche plus de l'équation 14 que l'équation 15.

Cependant, dans les calculs précédents, il n'a pas été tenu compte du fait que les essais au pressiomètre ont été effectués dans une cuve en acier de diamètre fini. Il est cependant possible d'estimer les effets :

- de l'épaisseur limitée de la glace se trouvant autour du pressiomètre :

- du confinement de la glace par la paroi de la cuve.

Selon FINNIE et HELLER (1959), la vitesse de déformation tangentielle $R / R$ à un rayon donné $R$ dans un tube épais soumis à un fluage stationnaire secondaire dû à une pression interne $\mathrm{p}$ est donnée par l'équation (1) :

$$
\begin{aligned}
& \frac{\dot{\mathrm{R}}}{\mathrm{R}}=\mathrm{A}\left(\frac{3}{4}\right)^{(\mathrm{n}+1) / 2} \\
& {\left[\frac{\mathrm{p}}{\left(\mathrm{R}_{\mathrm{e}} / \mathrm{R}_{i}\right)^{2 / \mathrm{n}}-1} \cdot \frac{2}{\mathrm{n}}\right]^{\mathrm{n}}\left(\frac{\mathrm{R}_{\mathrm{e}}}{\mathrm{R}}\right)^{2}}
\end{aligned}
$$

(1) Une équation identique utilisant une notation différente peut être trouvée dans un certain nombre de textes comme, par exemple, ODQVIST (1966). 
dans laquelle :

$\mathrm{R}_{\mathrm{i}}$ est le rayon intérieur du tube (dans notre cas, le rayon du forage);

$\mathrm{R}_{e}$ est le rayon extérieur du tube (dans notre cas, le rayon intérieur de la cuve) ;

$A$ et $n$ sont les valeurs $A$ et $n$ issues de la loi de GLEN pour le fluage secondaire en compression simple.

L'équation 16 suppose que la limite extérieure $\mathrm{R}_{e}$ est libre et soumise à une pression nulle.

Dans le cas des essais pressiométriques, le calcul de la vitesse de déformation tangentielle présente un intérêt le long de la paroi du forage lorsque $R=R_{1}=r$. L'équation 16 peut donc être réécrite sous la forme suivante :

$$
\frac{\dot{r}}{r}=D \cdot A \cdot p^{n}
$$

où :

$D=\left(\frac{3}{4}\right)^{(n+1) / 2}\left[\frac{\left(R_{e} / R_{i}\right)^{2 / n}}{\left(R_{e} / R_{i}\right)^{2 / n}-1} \cdot \frac{2}{n}\right]^{n}$

L'abaque de la figure 21 permet de déterminer $\mathrm{D}$ pour différentes combinaisons de $n$ et $R_{e} / R_{l}$.

Il faut noter que dans le cas des essais au pressiomètre en place, $R_{e} / R_{1} \rightarrow \infty$, et l'équation 18 , simplifiée, aboutit à :

$$
D=2^{-1}(3)^{(1+n) / 2} \cdot n^{-n}
$$

On remarque que, sous cette forme, $\mathrm{D}$ est l'inverse du terme qui relie $A$ et a dans l'équation 13.

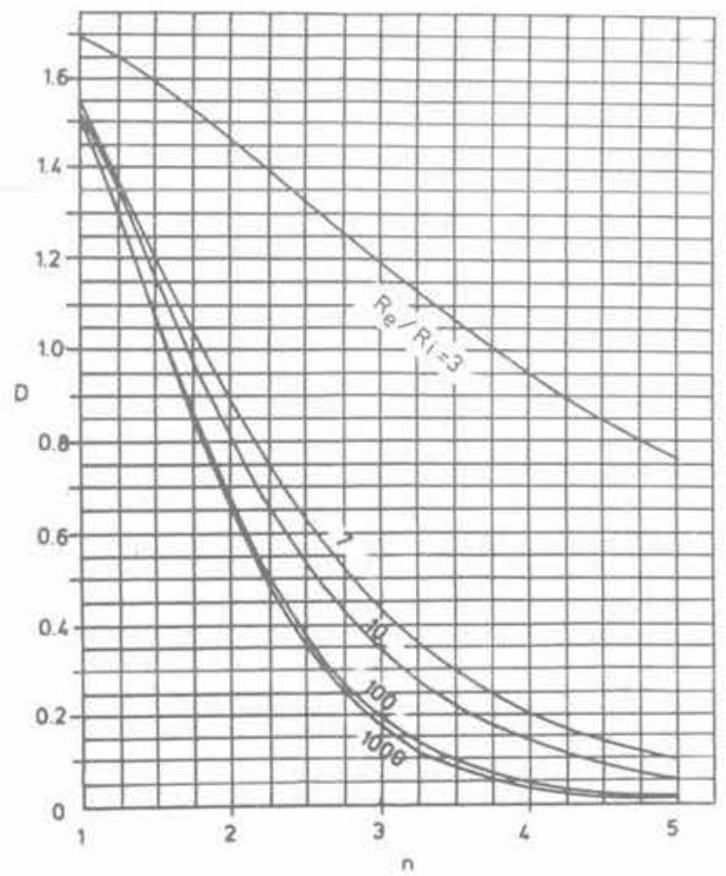

Fig. 21. - Fluage stationnaire au sein d'un cylindre épais : relation entre les parametres $D$ et $n$. Fig. 21. - Thick cylinder $D$ values.
Pour la valeur de n obtenue au pressiomètre (c'està-dire pour $n=3,76$ ) et pour les dimensions du tube en glace utilisé lors des essais pressiométriques (c'està-dire $R_{e}=445 \mathrm{~mm}$ et $R_{1}=38,5 \mathrm{~mm}$ ), la valeur de $\mathrm{D}$ est égale à 0,16 . Ainsi, nous obtenons :

$$
\frac{\dot{\mathrm{r}}}{\mathrm{r}}=0,16 \mathrm{Ap}^{3,76}
$$

La valeur de A ainsi obtenu pour $(\dot{\mathrm{r}} / \mathrm{r})_{\min }$ est de 2,4 $\times 10^{-7}$, ce qui permet une comparaison avec une valeur de $A$ de $8,2 \times 10^{-7}$ (équation 15) dans le cas des essais en place, quand $\mathrm{R}_{e}=\infty$ (voir aussi tableau 2).

Tableau 2. - Valeurs de $A$ et $n$

\begin{tabular}{|l|c|c|}
\hline \multicolumn{1}{|c|}{ Cas particuliers } & $\left.\mathrm{A}_{\mathrm{MPa}}\right)^{-\mathrm{n}_{\mathrm{s}}-1}$ & $\mathrm{n}$ \\
\hline $\begin{array}{l}\text { Compression simple } \\
\text { (à partir de Mellor } \\
\text { et Cole, 1982) }\end{array}$ & $4,3 \times 10^{-7}$ & 3,42 \\
\hline $\begin{array}{l}\text { Pressiomètre }\left(\mathrm{R}_{\mathrm{e}}=\infty,\right. \\
\text { régression linéaire) }\end{array}$ & $8,2 \times 10^{-7}$ & 3,76 \\
\hline $\begin{array}{l}\text { Pressiomètre }\left(\mathrm{R}_{\mathrm{e}}=\infty,\right. \\
\text { régression non linéaire) }\end{array}$ & $6,9 \times 10^{-7}$ & 3,46 \\
\hline $\begin{array}{l}\text { Pressiomètre (Tube épais, } \\
\mathrm{s}=0 \text { ) }\end{array}$ & $2,4 \times 10^{-7}$ & 3,76 \\
\hline
\end{tabular}

L'influence du confinement par les parois en acier de la cuve peut être prise en compte par une simple modification de l'équation 17. Selon LADANYI et ECKARDT (1983) le cas d'un tube épais confiné soumis à un fluage secondaire stationnaire peut être décrit par l'équation :

$$
\frac{\dot{\mathrm{r}}}{\mathrm{r}}=\mathrm{D} \cdot \mathrm{A} \cdot(\mathrm{p}-\mathrm{S})^{\mathrm{n}}
$$

où : $\mathrm{S}$ est la pression de confinement à l'extérieur du tube épais de glace.

Afin de calculer $S$ il est nécessaire de connaitre l'allongement des parois du tube. Puisque cette donnée n'a pas été mesurée dans nos essais, la déformation à la circonférence des parois d'acier d'une épaisseur de $11,7 \mathrm{~mm}$ doit être estimée à partir de l'augmentation de taille du forage. Cette estimation repose à son tour sur une hypothèse des caractéristiques de variation volumique de la glace, ce qui est une autre inconnue.

Si nous supposons que le volume de la glace ne change pas et que la glace ne flue pas verticalement dans la cuve, la valeur de $\mathrm{S}$, une fois que le diamètre de forage s'est agrandi de $4 \%$ (selon $(\dot{r} / r)_{\min }$ dans les essais au pressiomètre) est égale à 1,65 $\mathrm{MPa}$. Cette valeur élevée de $\mathrm{S}$ est tout à fait impossible puisque, si l'on se réfère à l'équation 21 , des essais au pressiomètre effectués à des valeurs de $1,0,1,25$ et $1,5 \mathrm{MPa}$ correspondraient à des valeurs négatives de $\mathrm{p}-\mathrm{S}$. 
Puisque la glace contenait des bulles d'air et qu'il n'y avait pas de confinement vertical des échantillons de glace (fig. 13) les hypothèses $n^{\circ} 1$ (pas de variation volumique) et $n^{\circ} 2$ (déformation plane) ne sont pas réalistes. Des études sont actuellement en cours afin d'analyser l'influence de ces deux phénomènes.

\subsection{Fluage primaire}

On peut maintenant aborder le problème de la représentation mathématique de la première portion des courbes de déformation, mesurée au pressiomètre, en fonction du temps. Selon LADANYI et JOHNSTON (1973), une relation simple entre le temps et la déformation (une loi " puissance ") (telle que décrite dans l'équation 5) peut être appliquée aux résultats d'essais de fluage au pressiomètre.

LADANYI et JOHNSTON proposent, à cet effet, une équation de la forme suivante:

$$
\ln \frac{\mathrm{r}}{\mathrm{r}_{\mathrm{o}}}=\mathrm{K} \cdot\left(\mathrm{p}-\mathrm{p}_{\mathrm{o}}\right)^{\mathrm{n}} \cdot \mathrm{t}^{\mathrm{b}}
$$

dans laquelle:

$\mathrm{K}$ est une constante dépendant du type de matériau et de la température,

$r$ est le rayon du forage au temps $t$;

$r_{\circ}$ est le rayon du forage au temps $t=0$;

$\mathrm{p}$ est la pression (constante) appliquée aux parois du forage ;

$\mathrm{p}_{\circ}$ est la pression s'exerçant loin du forage.

Il est à noter que, pour de faibles déformations :

$$
\ln \frac{\mathrm{r}}{\mathrm{r}_{\mathrm{o}}} \simeq \frac{\mathrm{r}-\mathrm{r}_{\mathrm{o}}}{\mathrm{r}_{\mathrm{o}}}=\frac{\Delta \mathrm{r}}{\mathrm{r}_{\mathrm{o}}}
$$

(déformation de la paroi du forage) ; $\left(\mathrm{p}-\mathrm{p}_{\mathrm{o}}\right)^{\mathrm{n}}$ étant une constante pour un essai effectué sous une pression donnée constante $\mathrm{p}$, l'équation 22 prendra la forme de l'équation 5 .

De ce qui précède, nous avons très souvent supposé que $p_{0}$ était nul. Cette hypothèse a été faite même lorsque les éprouvettes cylindriques de glace se trouvaient dans un conteneur rigide (acier). Une valeur non nulle de $\mathrm{p}_{\mathrm{o}}$ ne modifierait pas les conclusions de ce paragraphe puisque nous nous intéressons essentiellement au côté qualitatif du comportement de la glace.

La figure 22 regroupe les résultats des 8 essais pressiométriques, par le biais des variables $\log \left(\ln \frac{\mathrm{r}}{\mathrm{r}_{\mathrm{o}}}\right)$ et logt. La courbe correspondant à $\mathrm{p}=2 \mathrm{MPa}$ est retracée sous la forme d'une courbe en pointillé dans la figure 23 et un effort a été fait pour ajuster une ligne droite sur la première portion de cette courbe, c'est-à-dire avant la vitesse de déformation minimale (comme par ailleurs). Contrairement aux essais de compression simple pour lesquels on a trouvé qu'une loi puissance de la forme de l'équation 5 pouvait s'appliquer pratiquement tout du long jusqu'à la rupture (fig. 12), on ne peut, pour les essais pressiométriques, ajuster l'équation 22 que sur une portion très

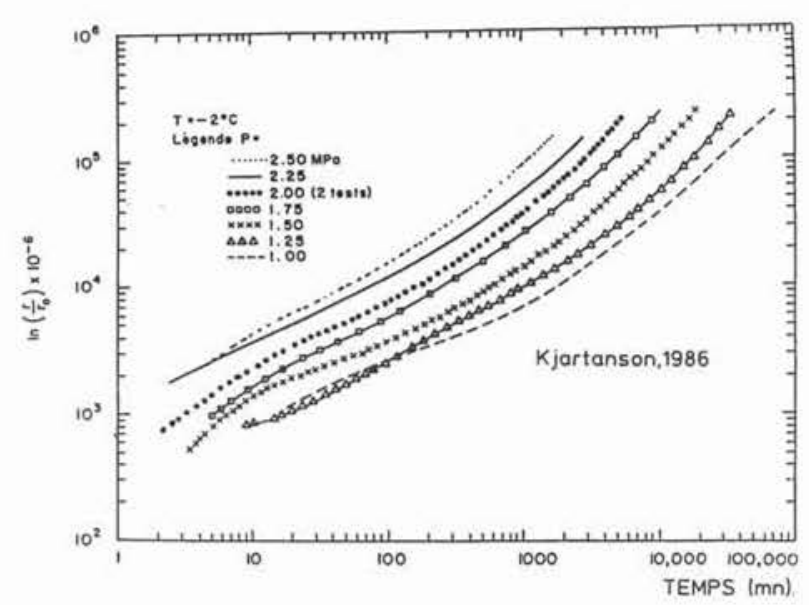

Fig. 22. - Evolution dans le temps des déformations lors d'essais de fluage au pressiomètre (cas de la glace pure). Fig. 22. - Strain versus time for constant pressure pressuremeter tests in ice.

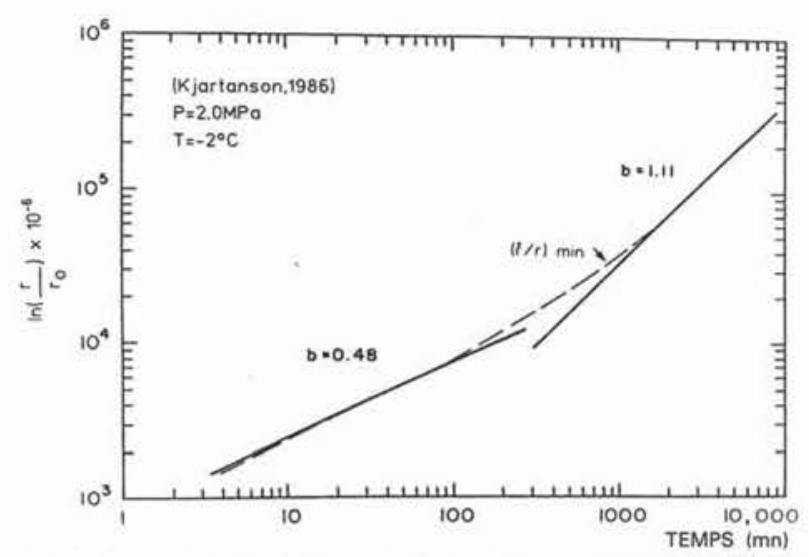

Fig. 23. - Analyse d'un essai de fluage au pressiomètre: non-représentativité d'une loi de puissance pour la description du fluage primaire.

Fig. 23. - Fitting a power law to primary creep during a constant pressure pressuremeter test.

réduite de la courbe expérimentale. Cette divergence signifie qu'une simple équation du type loi puissance (équation 22) ne s'applique pas au fluage primaire dans le cas du pressiomètre. L'écart constaté n'est probablement pas da au simple effet d'une redistribution de contraintes puisque l'ajustement est mauvais vers la fin de la période de fluage primaire ; en effet, la fin de la période de fluage primaire correspond à des temps suffisamment longs pour que l'on puisse raisonnablement supposer qu'une telle redistribution de contraintes soit achevée depuis longtemps.

L'équation 22 implique que :

- pour chaque valeur de la pression $\mathrm{p}$ appliquée par le pressiomètre, la courbe de fluage primaire reliant le logarithme népérien de $\frac{r}{r_{0}}$, (ln $\left.\frac{r}{r_{0}}\right)$, en fonction du temps, soit une droite dans $\stackrel{\mathrm{r}_{\mathrm{o}}}{\mathrm{r}}$ un diagramme bilogarithmique :

- que les droites correspondant aux différentes valeurs de la pression $\mathrm{p}$ soient des parallèles de pente commune $b$, dans ce même diagramme. 
Le lecteur pourra se référer à la figure 22 considérée sous ce nouvel angle de vue. Il semble en effet qu'on puisse faire un bon ajustement, avec des valeurs de b comprises entre 0,47 et 0,56 , sur les courbes correspondant à $\mathrm{p}=1,25,1,75,2,00,2,25$ et $2,50 \mathrm{MPa}$. D'autre part, des petites portions des courbes correspondant aux pressions $\mathrm{p}=1,00$ et $\mathrm{p}=$ $1,50 \mathrm{MPa}$ sont des segments de droite lorsque $\mathrm{b}$ est pris égal à 0,38 . Dans tous les cas, cependant, les parties linéarisables des courbes se terminent bien avant que le rapport $(\dot{r} / r)_{\min }$ correspondant à la rupture ait été atteint.

Pour l'instant, il n'est pas possible d'appliquer les équations de fluage primaire provenant des références suivantes au pressiomètre (GARDNER, JONES et HARRIS, 1984 ; SZYSZKOWSKI et GLOCKNER, 1985 ; ASHBY et DUVAL, 1985). Ces équations ne s'appliquent que pour des conditions aux limites représentées par des essais de compression classiques effectués sur les éprouvettes cylindriques.

MAN et al. (1985) et MAN et SUN (1986) font cependant état de progrès accomplis dans le cadre de leurs recherches quant à la détermination d'une loi de comportement traduisant le fluage primaire de la glace jusqu'à la rupture, loi qui s'appliquerait également au cas du pressiomètre. Jusqu'à présent, deux lois de comportement ont été proposées, qui sont toutes les deux des modèles de fluides de Rivlin-Ericksen de complexité 2. Pour une application au pressiomètre, ces modèles qui sont appelés respectivement fluide modifié du $2^{e}$ ordre (I) et fluide de complexité 2 à loi puissance (II) conduisent aux équations suivantes du fluage:

$$
\begin{gathered}
\mu_{2}(\ddot{\mathrm{r}} / \mathrm{r})+(\eta / \mathrm{m}+1)(\dot{\mathrm{r}} / \mathrm{r})^{\mathrm{m}+1} \\
-\frac{1}{2}\left(\mathrm{p}-\mathrm{p}_{\mathrm{o}}\right)=0 \\
\mu_{2}(\ddot{\mathrm{r}} / \mathrm{r})-(\mathrm{m} / \mathrm{m}+2) \mu_{2}(\dot{\mathrm{r}} / \mathrm{r})^{2}+\eta(\dot{\mathrm{r}} / \mathrm{r}) \\
-\frac{1}{2}\left(\mathrm{p}-\mathrm{p}_{\mathrm{o}}\right)(\mathrm{m}+1)(\dot{\mathrm{r}} / \mathrm{r})^{-\mathrm{m}}=0
\end{gathered}
$$

où : $\dot{\mathrm{r}}$ et $\ddot{\mathrm{r}}$ désignent respectivement la première et la deuxième dérivée du rayon de forage $r$ en fonction du temps et $\mu_{2}, \eta$ et $\mathrm{m}$ sont des paramètres caractéristiques du matériau. KJARTANSON (1986) et SUN (1987) font état du succès dans leurs tentatives d'obtenir les paramètres $\mu_{2}, \eta$ et $\mathrm{m}$ à partir des huit essais au pressiomètre mentionnés ci-dessus, et ce pour les deux modèles. MAN et SUN (1986) et SUN (1987) concluent que les deux modèles (I) et (II) fournissent des équations de fluage pour les essais triaxiaux en bonne concordance avec les résultats de McTIGUE et al., (1985) sur de la glace polycristalline à $-9,5^{\circ} \mathrm{C}$.

\section{CONCLUSIONS}

Il est tout à fait possible de réaliser des essais pressiométriques dans de la " glace chaude " pendant une durée pouvant aller jusqu'à 50 jours, tout en maintenant un degré de précision nécessaire sur la mesure de la déformation du forage.

Les résultats de déformation par fluage provenant des essais au pressiomètre effectués à pression constante peuvent être analysés de la même manière que les résultats d'essais de compression simple en laboratoire. Une analyse de ce type fournit la valeur de l'exposant $\mathrm{n}$ de la loi de Glen ainsi qu'un autre paramètre a permettant de calculer le paramètre $A$ de cette même loi.

Une simple représentation par une loi "puissance " du fluage primaire ne peut cependant pas être utilisée pour le pressiomètre, contrairement aux essais de compression. Sur une figure représentant le logarithme de la déformation en fonction du logarithme du temps, seule une petite portion de la courbe de déformation mesurée au pressiomètre est représentée sous forme de droite, par rapport à la linéarité pratiquement parfaite obtenue pour une représentation similaire de résultats d'essais de compression. Des recherches sont en cours afin de trouver une loi de fluage primaire pouvant s'appliquer au pressiomètre et permettant un ajustement avec les données présentées ci-dessus.

Cependant, la conclusion principale à retenir peut être que le pressiomètre permet de déterminer, en place, les paramètres du fluage des sols gelés et de la glace. Cela signifie qu'il existe donc une méthode qui permettra de surmonter tous les problèmes associés aux exigences d'échantillonnage et de manipulation de ces matériaux lorsque l'on effectue des essais en laboratoire. De plus, des essais au pressiomètre en place pourront être effectués dans des conditions réelles de température et de contrainte.

\section{REMERCIEMENTS}

Les essais au pressiomètre ont été effectués par KJARTANSON préparant son Doctorat à l'Université du Manitoba. Les subventions provenaient de l'Université et du Conseil de Recherches en Sciences Naturelles et en Génie du Canada. D'autres apports financiers ont été fournis par le North American Life Assurance Company, le Canada Mortgage and Housing Corporation et la OYO Corporation of Japan.

Parmi les nombreuses personnes qui ont contribué à cette recherche, nous remercions tout particulièrement MM. François BAGUELIN, Roger FRANK, Robert KENYON, Emery LAJTAI, Edward LEMKE et Brian TURNBULL. Philippe MESTAT a préparé la figure 21. Le texte a été traduit de l'anglais par S. PROESCHEL et B. SOYEZ.

\section{BIBLIOGRAPHIE}

ASHBY M.F., DUVAL P. (1985), The creep of polycrystalline ice. Cold Regions Science and Technology, 11, pp. 285-300.

BAGUELIN F., JÉZÉQUEL J.F., SHIELDS D.H. (1968). The pressuremeter and foundation engineering. Trans Tech. Publications, Clausthal, Germany, $617 \mathrm{p}$. 
FINNIE I., HELLER W.R. (1959), Creep of engineering materials. McGraw-Hill Book Company Inc., New York.

GARDNER A.R., JONES R.H., HARRIS J.S. (1984), A new creep equation for frozen soils and ice. Cold Regions Science and Technology, 9. pp. 271-275.

GLEN J.W. (1955), The creep of polycrystalline ice. Proceedings of the Royal Society, London, A 228 , pp. $519-538$.

JACKA T.H. (1984), The time and strain required for development of minimum strain rates in ice. Cold Regions Science and Technology, 8, pp. 261-268.

KJARTANSON B.H. (1986), Pressuremeter creep testing in laboratory ice. $\mathrm{Ph}$. D. thesis, University of Manitoba, Winnipeg, Manitoba, 400 p.

LADANYI B., JOHNSTON G.H. (1973), Evaluation of in situ creep properties of frozen soils with the pressuremeter. In : North American Contributions to the 2nd International Conference on Permafrost, Yakutsk, U.S.S.R, National Academy of Sciences, Washington, D.C., pp. 310-318.

MAN C.S., SHIELDS D.H., KJARTANSON B.H., SUN Q.X. (1985), Creep of ice as a fluid of complexity 2: the pressuremeter problem. Proceedings of the Tenth Canadian Congress of Applied Mechanics, University of Western Ontario, London, Ontario., pp. A347-A348.

MAN C.S., SUN Q.X. (1986), On the significance of normal stress effects in the flow of glaciers. IMA Preprint Series . 273, Institute for Mathematics and its Applications, University of Minnesota, Minneapolis, 26 p. Submitted to Journal of Glaciology.

McTIGUE D.F., PASSMAN S.L., JONES S.J. (1985), Normal stress effects in the creep of ice. Journal of Glaciology, 31, pp. 120-126.
MELLOR M. (1979), Mechanical properties of polycrystalline ice. In : Per Tryde (editor) Physicis and Mechanics of Ice, IUTAM Symposium, Copenhagen, Springer-Verlag, Berlin, pp. 217.245.

MELLOR M. COLE D.M. (1982), Deformation and failure of ice under constant stress or constant strain-rate. Cold Regions Science and Technology, 5, pp. 201-219.

MELLOR M., COLE D.M. (1983), Stress/strain/time relations for ice under uniaxial compression. Cold Regions Science and Technology, 6, pp. 207-230.

NYE J.F. (1957), The distribution of stress and velocity in glaciers and ice-sheets. Proceedings of the Royal Society, London, A 239, pp. 113-133.

ODQVIST F.K.G, (1966), Mathematical theory of creep and creep rupture. Oxford University Press, London, $170 \mathrm{p}$.

SEGO D.C. (1980), Deformation of ice under low stresses. Ph. D. thesis, University of Alberta, Edmonton, Alberta, 500 p.

SEGO D.C., MORGENSTERN N.R. (1983), Deformation of ice under low stresses. Canadian Geotechnical Journal, 20, pp. 587-602.

SUN Q.X. (1987), On two special Rivlin-Ericksen fluid models generalizing Glen's flow law for polycrys. talline ice. Ph. D. thesis, University of Manitoba. Winnipeg, Manitoba.

SZYSZKOWSKI W.. GLOCKNER P.G. (1985), Modelling the time-dependent behaviour of ice. Cold Regions Science and Technology, 11, p. 3-21.

VOYTKOVSKIY K.F. (1960), The mechanical properties of ice. Izvestia Akademia Nauk, Moscow, USSR. English translation AMS-T-R-391. United States Department of Commerce, Washington, D.C. 